\title{
III. BULK X-RAY MINERALOGY DATA
}

F. Mélières, University of Paris VI, France.

Measurements were made with an internal standard on material dried at $40^{\circ} \mathrm{C}$ and without further processing (such as washing in distilled water). The data are computed in per cent of the crystalline fraction of the sediment

Precision: $\pm 10 \% \quad \mathrm{~T}=<0.5 \%$.

Key to abbreviations on tables:

$\begin{array}{ll}\mathrm{Q} & =\text { quartz } \\ \mathrm{PIF} & =\text { plagioclase feldspars } \\ \mathrm{KF} & =\text { potassium feldspars } \\ \mathrm{C} & =\text { calcite (including Mg-calcite when it } \\ \mathrm{D} & =\text { occurs) } \\ & =\text { dolomite (including Ca-dolomite when it } \\ \mathrm{Ar} & =\text { aragonite }\end{array}$

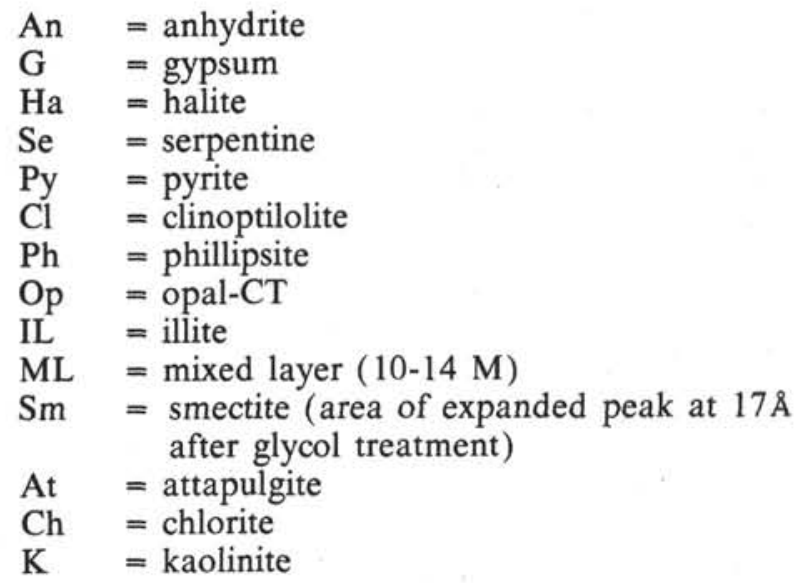

TABLE 1

Bulk X-Ray Mineralogy, Site 371

\begin{tabular}{|c|c|c|c|c|c|c|c|c|c|c|c|c|c|c|c|c|}
\hline $\begin{array}{c}\text { Sample } \\
\text { (Interval in } \mathrm{cm} \text { ) }\end{array}$ & Q & PIF & KF & $\mathrm{C}$ & D & $\mathrm{Ar}$ & G & An & $\mathrm{Ha}$ & IL & ML & $\mathrm{Sm}$ & At & $\mathrm{Ch}$ & $\mathrm{K}$ & Total \\
\hline $1-1,129-131$ & 18 & 3 & - & 28 & 1 & - & - & - & 1 & 21 & 15 & - & - & 7 & 7 & 100 \\
\hline $1-2,48-50$ & 14 & 2 & - & 29 & 1 & - & - & - & 1 & 25 & 16 & - & - & 9 & 3 & 100 \\
\hline $1-2,113-115$ & 18 & 3 & - & 20 & 1 & - & - & - & 1 & 21 & 24 & - & - & 6 & 6 & 100 \\
\hline $1-3,69-71$ & 14 & 2 & - & 29 & 2 & - & - & - & 2 & 22 & 17 & - & - & 7 & 5 & 100 \\
\hline $1-4,49-51$ & 21 & 5 & $\mathrm{~T}$ & 28 & 4 & - & - & - & 1 & 18 & 14 & - & - & 7 & 2 & 100 \\
\hline $1-5,44-46$ & 16 & 3 & $\mathrm{~T}$ & 22 & 3 & - & - & - & 1 & 25 & 18 & - & - & 9 & 4 & 100 \\
\hline $1-5,79-80$ & 16 & 3 & $\mathrm{~T}$ & 20 & 3 & - & - & - & 1 & 27 & 16 & - & - & 9 & 4 & 100 \\
\hline $1-6,45-47$ & 16 & 3 & $\mathrm{~T}$ & 14 & 2 & - & - & - & 1 & 31 & 19 & - & - & 9 & 5 & 100 \\
\hline $2-1,127-129$ & 18 & 5 & 1 & 19 & 2 & - & - & - & 2 & 30 & 10 & - & - & 9 & 4 & 100 \\
\hline $2-2,49-51$ & 15 & 3 & $\mathrm{~T}$ & 30 & 3 & - & - & - & 2 & 18 & 17 & - & - & 6 & 6 & 100 \\
\hline $2-3,49-51$ & 16 & 3 & 1 & 39 & 3 & - & - & - & 3 & 18 & 8 & - & - & 6 & 4 & 100 \\
\hline $3-1,79-81$ & 15 & 3 & 1 & 29 & 1 & - & - & - & 3 & 23 & 9 & - & - & 11 & 5 & 100 \\
\hline $3-2,49-51$ & 17 & 4 & 1 & 29 & 4 & - & - & - & 2 & 21 & 10 & - & - & 8 & 4 & 100 \\
\hline $3-3,49-51$ & 24 & 3 & 1 & 29 & 3 & - & - & - & 3 & 15 & 12 & - & - & 7 & 3 & 100 \\
\hline $3-4,47-49$ & 18 & 4 & 2 & 25 & 1 & - & - & - & 2 & 22 & 13 & - & - & 8 & 5 & 100 \\
\hline $3-5,50-52$ & 18 & 4 & 1 & 21 & 1 & - & - & - & 2 & 26 & 13 & - & - & 9 & 5 & 100 \\
\hline $3-6,49-51$ & 17 & 4 & $\mathrm{~T}$ & 25 & 2 & - & - & - & 2 & 21 & 15 & - & - & 9 & 5 & 100 \\
\hline $4-2,49-51$ & 19 & 4 & 1 & 28 & 3 & - & - & - & 3 & 18 & 10 & - & - & 8 & 6 & 100 \\
\hline $4-3,49-51$ & 18 & 6 & $\mathrm{~T}$ & 28 & 2 & - & - & - & 2 & 18 & 13 & - & - & 7 & 5 & 100 \\
\hline $4-4,48-50$ & 20 & 5 & 1 & 19 & 1 & - & - & - & 4 & 25 & 12 & - & - & 8 & 5 & 100 \\
\hline $4-5,49-51$ & 21 & 2 & 1 & 39 & 1 & - & - & - & 3 & 13 & 10 & - & - & 6 & 4 & 100 \\
\hline $4-6,46-47$ & 7 & 1 & 1 & 51 & 1 & - & - & - & 4 & 17 & 10 & - & - & 5 & 3 & 100 \\
\hline $4-6,92-94$ & 28 & 5 & 1 & 38 & 3 & - & - & - & 2 & 9 & 8 & - & - & 5 & 3 & 100 \\
\hline $5-1,36-38$ & 13 & 2 & $\mathrm{~T}$ & 43 & 2 & - & - & - & 3 & 19 & 9 & - & - & 6 & 3 & 100 \\
\hline $5-1,78-80$ & 21 & 2 & 1 & 36 & 3 & - & - & - & 2 & 16 & 8 & - & - & 6 & 5 & 100 \\
\hline $5-2,49-51$ & 13 & 4 & 2 & 32 & 2 & - & - & - & 3 & 23 & 10 & - & - & 7 & 4 & 100 \\
\hline $5-3,49-51$ & 10 & 2 & 1 & 37 & 1 & - & - & - & 3 & 20 & 14 & - & - & 7 & 5 & 100 \\
\hline $5-4,46-48$ & 16 & 3 & 1 & 26 & 3 & - & - & - & 3 & 26 & 12 & - & - & 8 & 2 & 100 \\
\hline $5-5,69-71$ & 15 & 4 & 1 & 26 & 3 & - & - & - & 2 & 26 & 12 & - & - & 9 & 2 & 100 \\
\hline $5-6,50-52$ & 17 & 4 & 2 & 26 & 5 & - & - & - & 2 & 17 & 14 & - & - & 7 & 6 & 100 \\
\hline $5, \mathrm{CC}$ & 15 & 3 & 2 & 23 & 5 & - & - & - & 2 & 26 & 11 & - & - & 9 & 4 & 100 \\
\hline $6, \mathrm{CC}$ & 11 & 2 & 1 & 31 & 3 & - & - & - & 3 & 25 & 12 & - & - & 7 & 6 & 100 \\
\hline $7, \mathrm{CC}$ & 15 & 1 & 1 & 46 & 2 & - & - & - & 3 & 14 & 9 & - & - & 5 & 4 & 100 \\
\hline $8-2,95-97$ & 27 & 9 & 2 & 11 & 16 & - & - & - & 3 & 10 & 9 & - & - & 9 & 4 & 100 \\
\hline $8-3,59-61$ & 25 & 7 & 3 & 15 & 13 & - & - & - & 2 & 9 & 11 & - & - & 11 & 4 & 100 \\
\hline
\end{tabular}


TABLE 2

Bulk X-Ray Mineralogy, Site 372

\begin{tabular}{|c|c|c|c|c|c|c|c|c|c|c|c|c|c|c|c|c|c|c|}
\hline $\begin{array}{c}\text { Sample } \\
\text { (Interval in } \mathrm{cm} \text { ) }\end{array}$ & Q & PIF & K F & C & D & Ar & G & An & $\mathrm{Ha}$ & $\mathrm{Cl}$ & Op & IL & ML & $\mathrm{Sm}$ & At & $\mathrm{Ch}$ & $\mathrm{K}$ & Total \\
\hline $1-1,128-130$ & 10 & 3 & 1 & 40 & 1 & - & - & - & 2 & - & - & 26 & 8 & - & - & 8 & 1 & 100 \\
\hline $1-2,53-55$ & 11 & 4 & 1 & 40 & 2 & - & - & - & 1 & - & - & 26 & 7 & - & - & 7 & 1 & 100 \\
\hline $1-3,49-51$ & 15 & 4 & 1 & 30 & 4 & - & - & - & 1 & - & - & 29 & 8 & - & - & 7 & 1 & 100 \\
\hline $1-4,40-41$ & 10 & 3 & 1 & 41 & 2 & - & - & - & 1 & - & - & 27 & 7 & - & - & 7 & 1 & 100 \\
\hline $2-1,129-132$ & 12 & 3 & 1 & 37 & 2 & - & - & - & 1 & - & - & 27 & 7 & - & - & 9 & 1 & 100 \\
\hline $2-2,45-47$ & 11 & 5 & 2 & 38 & 3 & - & - & - & 2 & - & - & 24 & 7 & - & - & 7 & 1 & 100 \\
\hline $2-3,50-51$ & 12 & 3 & 1 & 45 & 3 & - & - & - & 1 & - & - & 21 & 7 & - & - & 6 & 1 & 100 \\
\hline $2-4,11-13$ & 13 & 3 & 1 & 36 & 2 & - & 1 & - & 1 & - & - & 29 & 7 & - & - & 6 & 1 & 100 \\
\hline $2-4,64-66$ & 8 & 3 & 1 & 46 & 2 & - & - & - & 2 & - & - & 24 & 6 & - & - & 7 & 1 & 100 \\
\hline $3-1,70-72$ & 13 & 3 & 1 & 30 & 3 & - & - & - & 1 & - & - & 30 & 9 & - & - & 9 & 1 & 100 \\
\hline $3-2,50-52$ & 15 & 3 & 2 & 41 & 3 & - & - & - & 1 & - & - & 22 & 6 & - & - & 6 & 1 & 100 \\
\hline $3-3,50-52$ & 10 & 3 & 1 & 56 & 2 & - & - & - & 2 & - & - & 16 & 5 & - & - & 4 & 1 & 100 \\
\hline $4-1,103-105$ & 11 & 2 & 1 & 38 & 5 & - & - & - & 1 & - & - & 23 & 3 & 8 & - & 7 & 1 & 100 \\
\hline $4-2,50-52$ & 10 & 3 & 1 & 35 & 6 & - & 1 & - & 1 & - & - & 24 & 3 & 7 & - & 8 & 1 & 100 \\
\hline $5-1,137-139$ & 16 & 5 & 1 & 21 & 10 & - & $\mathbf{T}$ & - & 1 & - & - & 25 & 4 & 7 & - & 8 & 2 & 100 \\
\hline $6-1,145-147$ & 20 & 6 & 1 & 9 & 17 & - & - & - & 1 & - & - & 24 & 4 & 6 & - & 10 & 2 & 100 \\
\hline $9-1,127-129$ & 13 & 3 & 1 & 28 & 9 & - & - & - & 1 & - & - & 27 & 5 & 6 & - & 6 & 1 & 100 \\
\hline $9-2,69-71$ & 10 & 3 & 1 & 34 & 7 & - & - & - & 1 & - & - & 24 & 5 & 8 & - & 7 & - & 100 \\
\hline $9-3,60-62$ & 10 & 3 & 1 & 31 & 5 & - & - & - & 1 & - & - & 26 & 5 & 10 & - & 8 & - & 100 \\
\hline $9-4,50-52$ & 9 & 3 & 1 & 31 & 4 & - & - & - & 1 & - & - & 29 & 4 & 9 & - & 9 & - & 100 \\
\hline $9, \mathrm{CC}$ & 8 & 3 & 1 & 29 & 3 & - & - & - & 1 & - & - & 35 & 5 & 7 & - & 8 & - & 100 \\
\hline $10-1,100-102$ & 9 & 3 & 1 & 30 & 3 & - & - & - & 1 & - & - & 30 & 3 & 11 & - & 9 & - & 100 \\
\hline $10-2,80-82$ & 9 & 3 & 1 & 26 & 3 & - & - & - & 1 & - & - & 36 & 4 & 9 & - & 8 & - & 100 \\
\hline $11-1,100-102$ & 10 & 4 & 1 & 26 & 2 & - & - & - & 1 & - & - & 32 & 6 & 8 & - & 9 & 1 & 100 \\
\hline $11-2,50-52$ & 8 & 3 & 1 & 36 & 3 & - & $\mathrm{T}$ & - & 1 & - & - & 27 & 4 & 10 & - & 6 & 1 & 100 \\
\hline $11-3,50-52$ & 8 & 2 & 1 & 34 & 3 & - & 2 & - & 1 & - & - & 28 & 5 & 7 & - & 8 & 1 & 100 \\
\hline $12-1,20-22$ & 9 & 3 & 1 & 39 & 3 & - & - & - & 1 & - & - & 24 & 5 & 4 & - & 10 & 1 & 100 \\
\hline $12-3,50-52$ & 7 & 2 & $\mathrm{~T}$ & 43 & 3 & - & $\mathrm{T}$ & - & 1 & - & - & 25 & 4 & 3 & - & 10 & 1 & 100 \\
\hline $12-4,50-52$ & 8 & 2 & 1 & 46 & 2 & - & $\mathrm{T}$ & - & 1 & - & - & 25 & 4 & 3 & - & 7 & 1 & 100 \\
\hline $12-5,60-62$ & 6 & 3 & $\mathrm{~T}$ & 50 & 3 & - & $\mathrm{T}$ & - & 1 & - & - & 20 & 6 & 3 & - & 6 & 1 & 100 \\
\hline $12-6,50-52$ & 8 & 3 & $\mathrm{~T}$ & 44 & 2 & - & $\mathrm{T}$ & - & 1 & - & - & 24 & 6 & 3 & - & 8 & 1 & 100 \\
\hline $13-1,50-52$ & 7 & 2 & $\mathrm{~T}$ & 41 & 3 & - & $\mathrm{T}$ & - & 1 & - & - & 26 & 7 & 4 & - & 7 & 1 & 100 \\
\hline $13-2,50-52$ & 9 & 3 & 1 & 43 & 3 & - & $T$ & - & 1 & - & - & 22 & 6 & 5 & - & 6 & 1 & 100 \\
\hline $13-3,50-52$ & 8 & 3 & 1 & 37 & 3 & - & $\mathrm{T}$ & - & 1 & - & - & 34 & 5 & 4 & - & 4 & $\mathrm{~T}$ & 100 \\
\hline $13-4,50-52$ & 7 & 3 & 1 & 38 & 3 & - & - & - & 1 & - & - & 28 & 6 & 4 & - & 8 & 1 & 100 \\
\hline $13-5,50-52$ & 8 & 2 & $\mathrm{~T}$ & 50 & 3 & - & - & - & 1 & - & - & 21 & 3 & 5 & - & 6 & 1 & 100 \\
\hline $13-6,50-52$ & 7 & 2 & $\mathrm{~T}$ & 43 & 3 & - & - & - & 1 & - & - & 26 & 4 & 4 & - & 8 & 1 & 100 \\
\hline $14-1,50-52$ & 9 & 3 & $\mathrm{~T}$ & 41 & 3 & - & - & - & 1 & - & - & 27 & 3 & 5 & - & 9 & 1 & 100 \\
\hline $14-2,50-52$ & 9 & 2 & $\mathrm{~T}$ & 42 & 2 & - & - & - & 1 & - & - & 27 & 2 & 7 & - & 7 & 1 & 100 \\
\hline $14-3,50-52$ & 7 & 3 & 1 & 40 & 4 & - & - & - & 1 & - & - & 25 & 4 & 6 & - & 8 & 1 & 100 \\
\hline $14-4,50-52$ & 6 & 2 & 1 & 46 & 4 & - & - & - & 1 & - & - & 26 & 2 & 5 & - & 7 & 1 & 100 \\
\hline $14-5,50-52$ & 7 & 3 & 1 & 49 & 2 & - & - & - & 1 & - & - & 23 & 2 & 6 & - & 6 & - & 100 \\
\hline $14-6,60-62$ & 6 & 3 & 1 & 39 & 5 & - & - & - & 1 & - & - & 27 & 2 & 6 & - & 9 & 1 & 100 \\
\hline $15-1,30-32$ & 7 & 3 & $\mathrm{~T}$ & 51 & 4 & - & - & - & 1 & - & - & 20 & 3 & 6 & - & 5 & $\mathrm{~T}$ & 100 \\
\hline $15-2,50-52$ & 7 & 2 & $T$ & 48 & 6 & - & - & - & 1 & - & - & 23 & 2 & 5 & - & 6 & $\mathrm{~T}$ & 100 \\
\hline $15-3,30-32$ & 7 & 2 & $T$ & 47 & 3 & - & - & - & 1 & - & - & 23 & 3 & 6 & - & 6 & 1 & 100 \\
\hline $15-4,50-52$ & 8 & 3 & $T$ & 36 . & 5 & - & - & - & 1 & - & - & 27 & 4 & 6 & - & 9 & 1 & 100 \\
\hline $15-5,60-62$ & 9 & 3 & $\mathbf{T}$ & 42 & 4 & - & - & - & 1 & - & - & 20 & 5 & 9 & - & 7 & $\mathrm{~T}$ & 100 \\
\hline $15-6,50-52$ & 9 & 3 & 1 & 40 & 5 & - & - & - & 1 & - & - & 22 & 3 & 8 & - & 8 & - & 100 \\
\hline $16-1,60-62$ & 8 & 3 & $\mathrm{~T}$ & 38 & 3 & - & - & - & 1 & - & - & 29 & 3 & 7 & - & 8 & - & 100 \\
\hline $16-2,50-52$ & 8 & 3 & $T$ & 34 & 4 & - & - & - & 1 & - & - & 28 & 4 & 8 & - & 10 & - & 100 \\
\hline $16-3,50-52$ & 10 & 3 & $\mathrm{~T}$ & 37 & 3 & - & - & - & 1 & - & - & 25 & 3 & 12 & - & 7 & - & 100 \\
\hline $16-4,50-52$ & 10 & 4 & 1 & 35 & 4 & - & - & - & 2 & - & - & 26 & 3 & 7 & - & 8 & $\mathrm{~T}$ & 100 \\
\hline $16-5,60-62$ & 8 & 3 & 1 & 33 & 3 & - & - & - & 1 & - & - & 31 & 3 & 7 & - & 10 & - & 100 \\
\hline $16-6,48-50$ & 10 & 3 & 1 & 35 & 4 & - & - & - & 1 & - & - & 27 & 3 & 7 & - & 9 & $T$ & 100 \\
\hline $17-1,78-80$ & 8 & 4 & 1 & 39 & 4 & - & - & - & 2 & - & - & 27 & 2 & 4 & - & 8 & 1 & 100 \\
\hline $17-2,50-52$ & 7 & 3 & 1 & 35 & 4 & - & - & - & 1 & - & - & 31 & 3 & 6 & - & 9 & $\mathrm{~T}$ & 100 \\
\hline $17-3,50-52$ & 7 & 2 & 1 & 32 & 3 & - & - & - & 1 & - & - & 33 & 3 & 6 & - & 11 & 1 & 100 \\
\hline $17-4,50-52$ & 9 & 3 & $\mathrm{~T}$ & 38 & 4 & - & - & - & 1 & - & - & 27 & 2 & 7 & - & 8 & 1 & 100 \\
\hline $17-5,60-62$ & 9 & 3 & 1 & 41 & 4 & - & - & - & 2 & - & - & 24 & 1 & 7 & - & 8 & $T$ & 100 \\
\hline $17-6,50-52$ & 8 & 3 & $T$ & 40 & 4 & - & - & - & 1 & - & - & 30 & 1 & 5 & - & 8 & - & 100 \\
\hline
\end{tabular}


TABLE 2 - Continued

Sample

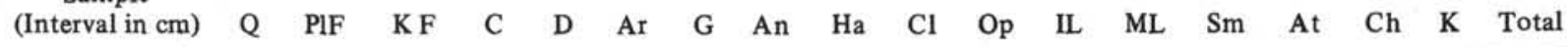

\begin{tabular}{|c|c|c|c|c|c|c|c|c|c|c|c|c|c|c|c|c|c|c|}
\hline $18-1,80-82$ & 8 & 3 & 1 & 43 & 3 & - & - & - & 1 & - & - & 27 & 1 & 4 & - & 9 & - & 100 \\
\hline $18-2,50-52$ & 8 & 3 & 1 & 40 & 3 & - & - & - & 1 & - & - & 29 & 2 & 4 & - & 9 & $\mathrm{~T}$ & 100 \\
\hline $18-3,50-52$ & 9 & 3 & 1 & 42 & 4 & - & - & - & 1 & - & - & 24 & 1 & 6 & - & 9 & - & 100 \\
\hline $18-4,50-52$ & 8 & 3 & 1 & 42 & 3 & - & - & - & 1 & - & - & 25 & 2 & 6 & - & 9 & - & 100 \\
\hline $18-5,50-52$ & 9 & 3 & 1 & 40 & 3 & - & - & - & 1 & - & - & 21 & 4 & 9 & - & 9 & - & 100 \\
\hline $18-6,50-52$ & 8 & 2 & $\mathrm{~T}$ & 43 & 3 & - & - & - & 1 & - & - & 23 & 3 & 9 & - & 8 & - & 100 \\
\hline $19-1,50-52$ & 7 & 2 & 1 & 43 & 2 & - & - & - & 2 & - & - & 27 & 1 & 6 & - & 9 & - & 100 \\
\hline $19-2,50-52$ & 6 & 2 & $\mathrm{~T}$ & 47 & 2 & - & - & - & 1 & - & - & 25 & 3 & 6 & - & 8 & - & 100 \\
\hline $19-3,50-52$ & 8 & 2 & $\mathrm{~T}$ & 43 & 3 & - & - & - & 1 & - & - & 24 & 2 & 9 & - & 8 & $\mathrm{~T}$ & 100 \\
\hline $19-4,50-52$ & 8 & 2 & $\mathrm{~T}$ & 42 & 3 & - & - & - & 2 & - & - & 23 & 2 & 11 & - & 7 & - & 100 \\
\hline $19-5,50-52$ & 8 & 3 & 1 & 37 & 2 & - & - & - & 2 & - & - & 26 & 3 & 10 & - & 8 & - & 100 \\
\hline $19-6,50-52$ & 6 & 2 & 1 & 47 & 3 & - & - & - & 1 & - & - & 22 & 2 & 9 & - & 7 & - & 100 \\
\hline $20-1,50-52$ & 7 & 3 & $\mathrm{~T}$ & 47 & 2 & - & - & - & 1 & - & - & 25 & 2 & 7 & - & 6 & $\mathrm{~T}$ & 100 \\
\hline $20-2,50-52$ & 6 & 2 & $\mathrm{~T}$ & 44 & 3 & - & - & _ & 1 & - & - & 27 & 2 & 8 & - & 7 & - & 100 \\
\hline $20-3,50-52$ & 7 & 3 & 1 & 50 & 3 & - & - & - & 1 & - & - & 19 & 1 & 9 & - & 6 & - & 100 \\
\hline $20-4,50-52$ & 6 & 3 & 1 & 45 & 4 & - & - & - & 1 & - & - & 24 & 2 & 7 & - & 7 & - & 100 \\
\hline $20-5,50-52$ & 6 & 2 & 1 & 46 & 3 & - & - & - & 1 & - & - & 25 & 3 & 6 & - & 7 & - & 100 \\
\hline $20-6,50-52$ & 8 & 4 & 1 & 35 & 3 & - & - & - & 2 & 1 & - & 29 & 4 & 5 & - & 8 & - & 100 \\
\hline $21-2,50-52$ & 6 & 2 & - & 52 & 3 & - & - & - & 1 & $T$ & - & 19 & 2 & 9 & - & 6 & - & 100 \\
\hline $21-3,48-50$ & 7 & 3 & - & 47 & 3 & - & - & - & 1 & 1 & - & 21 & 3 & 8 & - & 6 & $\mathrm{~T}$ & 100 \\
\hline $21-4,60-62$ & 6 & 2 & $T$ & 37 & 2 & - & - & - & 1 & $\mathrm{~T}$ & - & 29 & 4 & 10 & - & 8 & - & 100 \\
\hline $21-5,60-62$ & 7 & 3 & $\mathrm{~T}$ & 39 & 3 & - & - & - & 1 & 1 & - & 25 & 3 & 12 & - & 6 & $\mathrm{~T}$ & 100 \\
\hline $21-6,50-52$ & 7 & 2 & $T$ & 45 & 3 & - & - & - & 1 & 1 & - & 23 & 3 & 8 & - & 7 & - & 100 \\
\hline $22-1,50-52$ & 7 & 3 & $T$ & 44 & 3 & - & - & - & 1 & 2 & - & 22 & 3 & 8 & - & 7 & - & 100 \\
\hline $22-2,50-52$ & 7 & 2 & 2 & 43 & 3 & _ & - & _ & 1 & 1 & - & 23 & 3 & 9 & - & 6 & - & 100 \\
\hline $22-3,50-52$ & 7 & 3 & 1 & 42 & 3 & - & - & - & 1 & 1 & - & 24 & 3 & 9 & - & 7 & - & 100 \\
\hline $22-4,50-52$ & 7 & 2 & 1 & 50 & 3 & - & - & - & $\hat{1}$ & $\mathrm{~T}$ & - & 18 & 3 & 9 & - & 6 & $\mathrm{~T}$ & 100 \\
\hline $23-1,121-123$ & 7 & 2 & 2 & 43 & 3 & - & - & - & 1 & $\mathrm{~T}$ & - & 24 & 2 & 10 & - & 6 & - & 100 \\
\hline $23-2,50-52$ & 7 & 2 & 1 & 43 & 4 & _ & $\mathrm{T}$ & - & 1 & $\mathrm{~T}$ & - & 22 & 2 & 11 & - & 6 & - & 100 \\
\hline $23-3,50-52$ & 6 & 2 & 1 & 47 & 3 & - & - & - & 1 & - & - & 21 & 3 & 11 & - & 5 & - & 100 \\
\hline $23-4,60-62$ & 6 & 3 & 1 & 48 & 3 & _- & - & - & 1 & - & - & 20 & 3 & 8 & - & 7 & - & 100 \\
\hline $23-5,50-52$ & 5 & 2 & 1 & 48 & 2 & - & - & - & 1 & 1 & - & 23 & 2 & 9 & - & 6 & - & 100 \\
\hline $23-6,50-52$ & 6 & 2 & 1 & 51 & 3 & - & - & - & 1 & - & - & 20 & 2 & 11 & - & 5 & 1 & 100 \\
\hline $24-1,50-52$ & 6 & 2 & 1 & 42 & 3 & - & - & - & 1 & 1 & - & 26 & 3 & 9 & - & 6 & - & 100 \\
\hline $24-3,90-92$ & 8 & 3 & 2 & 47 & 2 & - & - & - & 2 & - & - & 17 & 3 & 9 & - & 6 & 1 & 100 \\
\hline $24-4,50-52$ & 7 & 2 & $\mathrm{~T}$ & 54 & 2 & - & - & - & 1 & - & - & 18 & 3 & 9 & - & 4 & $T$ & 100 \\
\hline $24-5,50-52$ & 7 & 2 & 1 & 52 & 2 & - & - & - & 1 & - & - & 18 & 2 & 9 & - & 5 & 1 & 100 \\
\hline $24-6,50-52$ & 5 & 1 & 1 & 58 & 2 & - & - & - & 1 & - & - & 18 & 3 & 6 & - & 5 & - & 100 \\
\hline $25-1,50-52$ & 7 & 1 & $\mathrm{~T}$ & 63 & 2 & - & - & - & 1 & - & - & 14 & 2 & 6 & - & 4 & - & 100 \\
\hline $25-2,50-52$ & 8 & 2 & $\mathrm{~T}$ & 44 & 2 & - & - & - & 2 & - & - & 27 & 3 & 5 & - & 6 & 1 & 100 \\
\hline $25-3,50-52$ & 8 & 2 & 1 & 51 & 2 & - & - & - & 3 & - & - & 18 & 4 & 4 & - & 6 & 1 & 100 \\
\hline $25-4,50-52$ & 6 & 1 & - & 51 & 2 & - & - & - & 1 & - & - & 24 & 5 & 3 & - & 7 & - & 100 \\
\hline $25-5,50-52$ & 5 & 1 & - & 68 & 2 & - & - & - & 1 & - & - & 13 & 1 & 4 & - & 4 & 1 & 100 \\
\hline $26-1,50-52$ & 7 & 1 & 1 & 49 & 2 & - & - & - & 2 & - & - & 22 & 3 & 6 & - & 7 & - & 100 \\
\hline $26-2,50-52$ & 6 & 1 & 1 & 61 & 2 & - & - & - & 1 & - & - & 16 & 2 & 4 & - & 5 & 1 & 100 \\
\hline $26-3,50-52$ & 7 & 2 & 1 & 44 & 3 & _ & _ & _ & 1 & - & - & 25 & 3 & 5 & - & 8 & 1 & 100 \\
\hline $26-4,50-52$ & 8 & 2 & $\mathrm{~T}$ & 45 & 3 & - & - & - & 1 & - & - & 20 & 5 & 8 & - & 7 & 1 & 100 \\
\hline $26-5,50-52$ & 9 & 2 & 1 & 42 & 3 & - & - & - & 2 & - & - & 22 & 4 & 6 & - & 8 & 1 & 100 \\
\hline $27-1,40-42$ & 6 & 1 & $\mathrm{~T}$ & 53 & 3 & - & - & - & 1 & - & - & 20 & 4 & 4 & - & 8 & - & 100 \\
\hline $27-2,50-52$ & 5 & 1 & 1 & 62 & 2 & - & - & - & 1 & - & - & 18 & 2 & 3 & - & 4 & 1 & 100 \\
\hline $27-3,50-52$ & 5 & 1 & 1 & 63 & 2 & - & - & - & 1 & - & - & 18 & 1 & 2 & - & 5 & 1 & 100 \\
\hline $27-4,50-52$ & 7 & 2 & 1 & 46 & 2 & - & - & - & 1 & - & - & 24 & 4 & 6 & - & 6 & 1 & 100 \\
\hline $27-5,50-52$ & 6 & 1 & 1 & 68 & 2 & - & - & - & 1 & - & - & 11 & 2 & 3 & - & 4 & 1 & 100 \\
\hline $28-1,100-102$ & 7 & 2 & $\mathrm{~T}$ & 47 & 2 & - & - & - & 2 & - & - & 22 & 3 & 9 & - & 5 & 1 & 100 \\
\hline $28-2,50-52$ & 6 & 1 & $\mathrm{~T}$ & 57 & 4 & _ & - & - & 1 & - & - & 16 & 3 & 7 & - & 4 & 1 & 100 \\
\hline $28-3,50-52$ & 6 & 2 & $\mathrm{~T}$ & 49 & 3 & - & - & - & 1 & - & - & 21 & 3 & 10 & - & 4 & 1 & 100 \\
\hline $28-4,50-52$ & 9 & 2 & 1 & 47 & 3 & - & - & _ & 1 & - & - & 20 & 2 & 12 & - & 4 & - & 100 \\
\hline $28-5,60-62$ & 6 & 2 & - & 55 & 2 & - & - & - & 1 & 2 & - & 16 & 3 & 9 & - & 4 & - & 100 \\
\hline $28-6,50-52$ & 10 & 9 & 4 & 34 & 5 & - & - & - & $\hat{1}$ & 5 & - & 15 & 2 & 10 & - & 4 & - & 100 \\
\hline $29-1,80-82$. & 9 & 2 & $\mathrm{~T}$ & 40 & 4 & - & - & - & 1 & 3 & - & 21 & 2 & 13 & - & 5 & - & 100 \\
\hline $29-2,50-52$ & 7 & 2 & 1 & 51 & 3 & _ & - & _ & 2 & 3 & - & 15 & 2 & 11 & - & 3 & - & 100 \\
\hline $29-3,50-52$ & 8 & 2 & 1 & 44 & 5 & - & $\mathrm{T}$ & - & 2 & 5 & - & 17 & 2 & 10 & - & 4 & - & 100 \\
\hline $29-4,50-52$ & 8 & 2 & $\mathrm{~T}$ & 31 & 4 & - & - & - & 1 & 2 & - & 25 & 3 & 18 & - & 5 & - & 100 \\
\hline $29-5,50-52$ & 8 & 1 & $\mathrm{~T}$ & 40 & 3 & - & - & - & 1 & 3 & - & 19 & 4 & 17 & - & 4 & - & 100 \\
\hline
\end{tabular}




\begin{tabular}{|c|c|c|c|c|c|c|c|c|c|c|c|c|c|c|c|c|c|c|}
\hline $\begin{array}{c}\text { Sample } \\
\text { (Interval in } \mathrm{cm} \text { ) }\end{array}$ & Q & PIF & K F & $\mathrm{C}$ & D & $\mathrm{Ar}$ & G & An & $\mathrm{Ha}$ & $\mathrm{Cl}$ & Op & IL & ML & $\mathrm{Sm}$ & At & $\mathrm{Ch}$ & $\mathrm{K}$ & Total \\
\hline $30-2,50-52$ & 8 & 2 & $\mathrm{~T}$ & 43 & 1 & - & - & - & 2 & - & - & 16 & 1 & 23 & - & 4 & - & 100 \\
\hline $30-3,50-52$ & 6 & 2 & 1 & 39 & 5 & - & - & - & 1 & 5 & - & 21 & 2 & 14 & - & 4 & - & 100 \\
\hline $30-4,50-52$ & 6 & 2 & 1 & 42 & 3 & - & - & - & 1 & 5 & - & 18 & 2 & 16 & - & 4 & - & 100 \\
\hline $30-5,50-52$ & 8 & 2 & $\mathrm{~T}$ & 48 & 3 & - & - & - & 2 & 4 & - & 13 & 2 & 14 & - & 4 & - & 100 \\
\hline $30-6,51-53$ & 6 & 1 & $\mathrm{~T}$ & 54 & 3 & - & - & - & 1 & 6 & - & 14 & 2 & 10 & - & 3 & - & 100 \\
\hline $31-1,105-107$ & 8 & 2 & - & 39 & 5 & - & - & - & 1 & 8 & - & 16 & 2 & 15 & - & 4 & - & 100 \\
\hline $31-2,50-52$ & 7 & 2 & - & 36 & 6 & - & - & - & 1 & 6 & - & 23 & 1 & 12 & - & 6 & - & 100 \\
\hline $31-3,43-44$ & 7 & 2 & 1 & 45 & 3 & - & - & - & 1 & 2 & - & 17 & 2 & 16 & - & 4 & - & 100 \\
\hline $31-4,26-28$ & 7 & 2 & $\mathrm{~T}$ & 50 & 5 & _ & - & - & 1 & 4 & _ & 12 & 3 & 13 & - & 3 & - & 100 \\
\hline $31-5,50-52$ & 7 & 2 & - & 49 & 5 & - & - & - & 1 & 5 & - & 17 & 1 & 9 & - & 4 & _- & 100 \\
\hline $31-6,29-31$ & 8 & 2 & - & 41 & 5 & - & - & - & 1 & 8 & - & 18 & 2 & 10 & - & 5 & - & 100 \\
\hline $32-1,50-52$ & 8 & 2 & - & 54 & 3 & - & - & - & 1 & 10 & - & 10 & 1 & 8 & - & 3 & - & 100 \\
\hline $32-3,70-72$ & 5 & 2 & - & 67 & 2 & - & - & - & 1 & 1 & - & 10 & 2 & 7 & - & 3 & - & 100 \\
\hline $32-4,57-59$ & 8 & 2 & $\mathrm{~T}$ & 41 & 6 & - & - & - & 1 & 8 & - & 18 & 2 & 9 & - & 5 & - & 100 \\
\hline $32-5,45-47$ & 8 & 2 & - & 50 & 5 & - & - & - & 1 & 5 & - & 14 & 2 & 10 & - & 3 & - & 100 \\
\hline $32-6,60-63$ & 8 & 3 & 1 & 34 & 6 & - & - & - & 1 & 9 & - & 18 & 3 & 12 & - & 5 & - & 100 \\
\hline $33-1,37-39$ & 7 & 3 & $\mathrm{~T}$ & 56 & 6 & - & - & - & 1 & 5 & - & 12 & 1 & $6^{\circ}$ & - & 3 & - & 100 \\
\hline $33-2,50-52$ & 5 & 1 & $\mathrm{~T}$ & 65 & 5 & - & - & - & 2 & 8 & - & 7 & 1 & 3 & - & 2 & - & 100 \\
\hline $33-3,40-42$ & 4 & 1 & - & 69 & 3 & - & - & - & 1 & 3 & - & 7 & 3 & 7 & - & 2 & - & 100 \\
\hline $33-4,75-77$ & 10 & 3 & - & 32 & 3 & - & - & - & 1 & 3 & - & 24 & 4 & 11 & - & 9 & - & 100 \\
\hline $33-5,60-62$ & 10 & 4 & - & 26 & 3 & - & $\mathrm{T}$ & - & 1 & 2 & - & 30 & 2 & 12 & - & 10 & - & 100 \\
\hline $33-6,71-72$ & 13 & 5 & 1 & 29 & 4 & - & $\mathrm{T}$ & - & 1 & 2 & - & 22 & 3 & 12 & - & 8 & - & 100 \\
\hline $34-1,127-129$ & 10 & 4 & 1 & 20 & 14 & - & - & - & 1 & - & 6 & 24 & 2 & 6 & - & 12 & - & 100 \\
\hline $34-2,42-44$ & 12 & 4 & 1 & 20 & 7 & - & $\mathrm{T}$ & - & 1 & - & 10 & 21 & 3 & 10 & - & 11 & - & 100 \\
\hline $34-3,69-71$ & 15 & 4 & 1 & 19 & 5 & - & $\mathrm{T}$ & - & 1 & - & 6 & 21 & 3 & 14 & - & 11 & - & 100 \\
\hline $34-4,28-30$ & 10 & 3 & 1 & 27 & 4 & - & - & - & 1 & - & 5 & 24 & 3 & 12 & - & 10 & - & 100 \\
\hline $34-5,15-17$ & 14 & 4 & 1 & 26 & 4 & - & - & - & 1 & - & 4 & 18 & 4 & 16 & - & 8 & - & 100 \\
\hline $35-1,102-105$ & 12 & 4 & 1 & 26 & 5 & - & $\mathrm{T}$ & - & 1 & - & 10 & 20 & 3 & 7 & - & 11 & - & 100 \\
\hline $35-2,30-32$ & 12 & 5 & 1 & 19 & 5 & - & 1 & - & 1 & - & 10 & 24 & 4 & 7 & - & 11 & - & 100 \\
\hline $35-3,67-69$ & 13 & 4 & 1 & 21 & 4 & - & $\mathrm{T}$ & - & 1 & - & 8 & 20 & 5 & 9 & - & 14 & - & 100 \\
\hline $36-1,30-32$ & 13 & 5 & 2 & 25 & 4 & - & - & - & 1 & - & 8 & 20 & 3 & 10 & - & 9 & - & 100 \\
\hline $36-2,31-33$ & 13 & 4 & 1 & 22 & 4 & - & - & - & 1 & - & 5 & 27 & 3 & 9 & - & 11 & - & 100 \\
\hline $36-3,50-52$ & 13 & 6 & 1 & 22 & 4 & - & - & - & 1 & - & 8 & 25 & 2 & 6 & - & 12 & - & 100 \\
\hline $36-4,55-58$ & 15 & 5 & 1 & 20 & 5 & - & - & - & 1 & - & 7 & 22 & 3 & 9 & - & 12 & - & 100 \\
\hline $36-5,31-33$ & 13 & 5 & 1 & 18 & 8 & - & $\mathrm{T}$ & - & 1 & - & 6 & 23 & 3 & 9 & - & 13 & - & 100 \\
\hline $36-6,41-43$ & 14 & 5 & 1 & 23 & 10 & - & - & - & 1 & - & $\mathrm{T}$ & 22 & 3 & 10 & - & 11 & - & 100 \\
\hline $37-1,132-135$ & 11 & 4 & 1 & 23 & 2 & - & - & - & 1 & - & 5 & 21 & 3 & 19 & - & 10 & - & 100 \\
\hline $37-2,41-43$ & 13 & 4 & 1 & 25 & 4 & - & - & - & 1 & - & 6 & 20 & 3 & 11 & - & 11 & - & 100 \\
\hline $37-3,74-76$ & 13 & 4 & 1 & 22 & 3 & - & $\mathrm{T}$ & - & 1 & - & 6 & 23 & 3 & 13 & - & 11 & - & 100 \\
\hline $37-4,58-60$ & 14 & 4 & 1 & 19 & 3 & - & - & - & 1 & - & 4 & 22 & 3. & 17 & - & 12 & - & 100 \\
\hline $38-1,44-46$ & 11 & 3 & 1 & 21 & 11 & - & - & - & 1 & - & 12 & 20 & 2 & 9 & - & 9 & - & 100 \\
\hline $38-2,40-42$ & 14 & 5 & 1 & 20 & 7 & - & - & - & 1 & - & 7 & 24 & 2 & 10 & - & 10 & - & 100 \\
\hline $38-3,50-52$ & 13 & 5 & $\mathrm{~T}$ & 21 & 7 & - & - & - & 1 & - & 10 & 19 & 2 & 11 & - & 11 & - & 100 \\
\hline $38-4,48-50$ & 12 & 4 & 1 & 19 & 7 & - & - & - & 1 & - & 11 & 23 & 2 & 8 & - & 12 & - & 100 \\
\hline $38-5,55-57$ & 13 & 5 & $\mathrm{~T}$ & 17 & 12 & - & - & - & 1 & - & 8 & 18 & 3 & 13 & - & 9 & - & 100 \\
\hline $38-6,118-120$ & 12 & 5 & 1 & 19 & 11 & - & - & - & 1 & - & 2 & 25 & 3 & 11 & - & 10 & - & 100 \\
\hline $39-1,36-38$ & 11 & 4 & 1 & 26 & 4 & - & 1 & - & 1 & - & 8 & 23 & 3 & 10 & - & 8 & - & 100 \\
\hline $39-2,58-62$ & 15 & 6 & $\mathrm{~T}$ & 27 & 4 & - & - & - & 1 & - & 1 & 22 & 3 & 11 & - & 9 & - & 100 \\
\hline $39-3,58-62$ & 10 & 4 & 1 & 21 & 4 & - & - & - & 1 & - & 8 & 24 & 4 & 15 & - & 8 & - & 100 \\
\hline $39-4,96-99$ & 11 & 3 & 1 & 22 & 7 & - & - & - & 1 & - & 17 & 17 & 5 & 9 & - & 7 & - & 100 \\
\hline $39-5,62-64$ & 11 & 5 & 1 & 19 & 15 & - & $\mathrm{T}$ & - & 1 & - & 8 & 21 & 4 & 9 & - & 6 & - & 100 \\
\hline $39-6,54-56$ & 11 & 4 & 1 & 19 & 11 & - & $\mathrm{T}$ & - & 1 & - & 15 & 21 & 2 & 7 & - & 8 & - & 100 \\
\hline $40-1,120-122$ & 11 & 5 & 1 & 17 & 17 & - & - & - & 1 & - & 8 & 23 & 2 & 7 & - & 8 & - & 100 \\
\hline $40-2,11-13$ & 13 & 6 & 1 & 18 & 16 & - & - & - & 1 & - & 8 & 17 & 3 & 8 & - & 9 & - & 100 \\
\hline $40-3,54-56$ & 12 & 4 & 1 & 15 & 13 & - & $\mathrm{T}$ & - & 1 & - & 10 & 22 & 3 & 8 & - & 11 & _ & 100 \\
\hline $40-4,90-92$ & 11 & 4 & 1 & 18 & 7 & - & $\mathrm{T}$ & - & 1 & - & 18 & 23 & 3 & 8 & - & 6 & - & 100 \\
\hline $40-5,103-104$ & 12 & 5 & 1 & 22 & 4 & - & - & - & 1 & - & 7 & 24 & 4 & 11 & - & 9 & - & 100 \\
\hline $40-6,69-71$ & 14 & 5 & 1 & 24 & 5 & - & $\mathrm{T}$ & - & $\hat{1}$ & - & 2 & 25 & 3 & 11 & - & 9 & - & 100 \\
\hline $41-1,64-65$ & 14 & 5 & 2 & 19 & 4 & - & - & - & 1 & - & 12 & 22 & 2 & 9 & - & 10 & - & 100 \\
\hline $41-2,60-62$ & 12 & 5 & 1 & 20 & 3 & - & $\mathrm{T}$ & - & 1 & - & 11 & 26 & 3 & 8 & - & 10 & - & 100 \\
\hline $41-3,79-81$ & 12 & 6 & 1 & 19 & 3 & - & $\mathrm{T}$ & - & 1 & - & 8 & 28 & 3 & 5 & - & 14 & - & 100 \\
\hline $41-4,56-58$ & 14 & 5 & 2 & 24 & 4 & - & $\mathrm{T}$ & - & 1 & - & 8 & 23 & 2 & 7 & - & 10 & - & 100 \\
\hline $41-5,72-74$ & 14 & 7 & 2 & 24 & 3 & - & - & - & 1 & - & - & 26 & 2 & 11 & - & 10 & - & 100 \\
\hline $41-6,64-66$ & 12 & 5 & 1 & 23 & 4 & - & - & - & 1 & - & 5 & 26 & 2 & 11 & - & 10 & - & 100 \\
\hline
\end{tabular}


TABLE 2 - Continued

\begin{tabular}{|c|c|c|c|c|c|c|c|c|c|c|c|c|c|c|c|c|c|c|}
\hline $\begin{array}{c}\text { Sample } \\
\text { (Interval in } \mathrm{cm} \text { ) }\end{array}$ & Q & PIF & K F & C & D & $\mathrm{Ar}$ & G & An & $\mathrm{Ha}$ & $\mathrm{Cl}$ & Op & IL & ML & $\mathrm{Sm}$ & At & $\mathrm{Ch}$ & $\mathrm{K}$ & Total \\
\hline $\begin{array}{l}42-1,60-62 \\
42-2,67-69 \\
42-3,68-70 \\
42-4,47-49 \\
42-5,61-64 \\
42-6,52-54\end{array}$ & $\begin{array}{l}12 \\
11 \\
12 \\
12 \\
13 \\
13\end{array}$ & $\begin{array}{l}5 \\
4 \\
6 \\
5 \\
6 \\
5\end{array}$ & $\begin{array}{l}1 \\
1 \\
1 \\
\mathrm{~T} \\
\mathrm{~T} \\
\mathrm{~T}\end{array}$ & $\begin{array}{l}18 \\
20 \\
19 \\
22 \\
20 \\
21\end{array}$ & $\begin{array}{l}3 \\
4 \\
4 \\
4 \\
3 \\
3 \\
3\end{array}$ & $\begin{array}{l}- \\
- \\
- \\
- \\
-\end{array}$ & $\begin{array}{l}- \\
- \\
- \\
- \\
-\end{array}$ & $\begin{array}{l}- \\
\overline{-} \\
\overline{-} \\
\overline{-}\end{array}$ & $\begin{array}{l}2 \\
1 \\
1 \\
1 \\
1 \\
1\end{array}$ & $\begin{array}{l}- \\
- \\
- \\
- \\
-\end{array}$ & $\begin{array}{r}7 \\
9 \\
11 \\
10 \\
7 \\
4\end{array}$ & $\begin{array}{l}28 \\
27 \\
27 \\
25 \\
25 \\
31\end{array}$ & $\begin{array}{l}3 \\
2 \\
2 \\
2 \\
3 \\
2\end{array}$ & $\begin{array}{r}10 \\
9 \\
7 \\
11 \\
12 \\
9\end{array}$ & $\begin{array}{l}- \\
- \\
- \\
- \\
-\end{array}$ & $\begin{array}{r}11 \\
12 \\
10 \\
8 \\
10 \\
11\end{array}$ & $\begin{array}{l}- \\
- \\
- \\
- \\
-\end{array}$ & $\begin{array}{l}100 \\
100 \\
100 \\
100 \\
100 \\
100\end{array}$ \\
\hline $\begin{array}{l}43-2,71-73 \\
43-3,62-64 \\
43-4,99-101 \\
43-5,63-66 \\
43-6,103-104\end{array}$ & $\begin{array}{l}12 \\
13 \\
11 \\
14 \\
12\end{array}$ & $\begin{array}{l}5 \\
5 \\
4 \\
5 \\
5\end{array}$ & $\begin{array}{l}1 \\
1 \\
1 \\
\mathrm{~T} \\
1\end{array}$ & $\begin{array}{l}22 \\
26 \\
23 \\
24 \\
21\end{array}$ & $\begin{array}{l}4 \\
4 \\
4 \\
4 \\
3\end{array}$ & $\begin{array}{l}- \\
- \\
- \\
-\end{array}$ & $\begin{array}{l}- \\
- \\
- \\
T\end{array}$ & $\begin{array}{l}- \\
- \\
- \\
-\end{array}$ & $\begin{array}{l}1 \\
1 \\
1 \\
1 \\
1\end{array}$ & $\begin{array}{l}- \\
- \\
- \\
-\end{array}$ & $\begin{array}{l}2 \\
1 \\
3 \\
1 \\
2\end{array}$ & $\begin{array}{l}32 \\
26 \\
30 \\
29 \\
31\end{array}$ & $\begin{array}{l}2 \\
3 \\
2 \\
2 \\
3\end{array}$ & $\begin{array}{r}8 \\
9 \\
11 \\
10 \\
10\end{array}$ & $\begin{array}{l}- \\
- \\
- \\
-\end{array}$ & $\begin{array}{l}11 \\
11 \\
10 \\
10 \\
11\end{array}$ & $\begin{array}{l}- \\
- \\
- \\
-\end{array}$ & $\begin{array}{l}100 \\
100 \\
100 \\
100 \\
100\end{array}$ \\
\hline $\begin{array}{l}44-1,36-38 \\
44-2,30-32 \\
44-3,97-99 \\
44-4,50-52 \\
44-5,84-86 \\
44-6,61-63\end{array}$ & $\begin{array}{l}12 \\
14 \\
13 \\
27 \\
28 \\
14\end{array}$ & $\begin{array}{l}4 \\
5 \\
7 \\
9 \\
7 \\
5\end{array}$ & $\begin{array}{l}1 \\
1 \\
1 \\
\mathrm{~T} \\
\mathrm{~T} \\
1\end{array}$ & $\begin{array}{l}20 \\
21 \\
23 \\
20 \\
24 \\
24\end{array}$ & $\begin{array}{r}3 \\
4 \\
4 \\
32 \\
20 \\
4\end{array}$ & $\begin{array}{l}- \\
- \\
- \\
- \\
-\end{array}$ & $\begin{array}{l}- \\
- \\
- \\
\bar{T} \\
T\end{array}$ & $\begin{array}{l}- \\
- \\
- \\
-\end{array}$ & $\begin{array}{l}1 \\
1 \\
1 \\
1 \\
1 \\
1 \\
1\end{array}$ & $\begin{array}{l}- \\
- \\
- \\
- \\
-\end{array}$ & $\begin{array}{l}8 \\
2 \\
2 \\
- \\
- \\
2\end{array}$ & $\begin{array}{r}31 \\
28 \\
28 \\
8 \\
13 \\
27\end{array}$ & $\begin{array}{l}2 \\
2 \\
2 \\
- \\
1 \\
2\end{array}$ & $\begin{array}{r}8 \\
12 \\
10 \\
1 \\
2 \\
11\end{array}$ & $\begin{array}{l}- \\
- \\
- \\
- \\
-\end{array}$ & $\begin{array}{r}10 \\
10 \\
9 \\
2 \\
4 \\
9\end{array}$ & $\begin{array}{l}- \\
- \\
- \\
\overline{-} \\
-\end{array}$ & $\begin{array}{l}100 \\
100 \\
100 \\
100 \\
100 \\
100\end{array}$ \\
\hline $\begin{array}{l}45-1,86-88 \\
45-2,28-30 \\
45-3,46-48 \\
45-4,76-78 \\
45-5,75-77 \\
45-6,84-86\end{array}$ & $\begin{array}{l}16 \\
18 \\
14 \\
15 \\
14 \\
14\end{array}$ & $\begin{array}{l}8 \\
5 \\
4 \\
4 \\
4 \\
4\end{array}$ & $\begin{array}{l}1 \\
1 \\
\mathrm{~T} \\
1 \\
\mathrm{~T} \\
\mathrm{~T}\end{array}$ & $\begin{array}{l}25 \\
14 \\
18 \\
18 \\
19 \\
19\end{array}$ & $\begin{array}{r}12 \\
10 \\
5 \\
8 \\
5 \\
4\end{array}$ & $\begin{array}{l}- \\
- \\
- \\
- \\
-\end{array}$ & $\begin{array}{l}- \\
- \\
- \\
- \\
-\end{array}$ & $\begin{array}{l}- \\
- \\
- \\
- \\
-\end{array}$ & $\begin{array}{l}1 \\
1 \\
1 \\
1 \\
1 \\
1\end{array}$ & $\begin{array}{l}- \\
- \\
- \\
- \\
-\end{array}$ & $\begin{array}{l}5 \\
6 \\
3 \\
5 \\
7 \\
9\end{array}$ & $\begin{array}{l}19 \\
27 \\
36 \\
31 \\
31 \\
29\end{array}$ & $\begin{array}{l}1 \\
1 \\
2 \\
2 \\
2 \\
2\end{array}$ & $\begin{array}{r}5 \\
10 \\
9 \\
7 \\
9 \\
10\end{array}$ & $\begin{array}{l}- \\
- \\
- \\
- \\
-\end{array}$ & $\begin{array}{l}7 \\
7 \\
8 \\
8 \\
8 \\
8\end{array}$ & $\begin{array}{l}- \\
\overline{-} \\
- \\
\overline{-} \\
-\end{array}$ & $\begin{array}{l}100 \\
100 \\
100 \\
100 \\
100 \\
100\end{array}$ \\
\hline $\begin{array}{l}46-2,84-86 \\
46-3,63-65\end{array}$ & $\begin{array}{l}15 \\
14\end{array}$ & $\begin{array}{l}5 \\
5\end{array}$ & $\begin{array}{l}1 \\
\mathrm{~T}\end{array}$ & $\begin{array}{l}21 \\
19\end{array}$ & $\begin{array}{l}9 \\
8\end{array}$ & - & - & - & $\begin{array}{l}1 \\
1\end{array}$ & - & $\begin{array}{l}7 \\
5\end{array}$ & $\begin{array}{l}23 \\
31\end{array}$ & $\begin{array}{l}2 \\
2\end{array}$ & $\begin{array}{l}9 \\
8\end{array}$ & - & $\begin{array}{l}7 \\
7\end{array}$ & - & $\begin{array}{l}100 \\
100\end{array}$ \\
\hline
\end{tabular}

TABLE 3

Bulk X-Ray Mineralogy, Site 373A

\begin{tabular}{lrrrrrrrrrrrrrrrrr}
\hline $\begin{array}{c}\text { Sample } \\
\text { (Interval in cm) }\end{array}$ & Q & PIF & KF & C & D & Ar & G & An & Ha & Ph & IL & ML & Sm & At & Ch & K & Total \\
\hline $1-1,48-50$ & 21 & 15 & 9 & 21 & 5 & - & - & - & 3 & - & 15 & 4 & 2 & - & 4 & 1 & 100 \\
$1-1,64-66$ & 10 & 4 & 1 & 46 & 1 & - & - & - & 2 & - & 17 & 5 & 6 & - & 4 & 4 & 100 \\
$1-1,88-90$ & 11 & 4 & 1 & 32 & 1 & - & - & - & 2 & 14 & 19 & 6 & 4 & - & 3 & 3 & 100 \\
$1-2,8-10$ & 11 & 4 & 2 & 28 & 1 & - & - & - & 3 & 9 & 17 & 7 & 10 & - & 4 & 4 & 100 \\
$1-2,143-145$ & 10 & 3 & 1 & 32 & - & - & - & - & 2 & - & 25 & 4 & 8 & - & 7 & 8 & 100 \\
$1-3,51-53$ & 14 & 6 & 1 & 24 & 3 & - & - & - & 2 & - & 27 & 4 & 8 & - & 6 & 6 & 100 \\
$1-4,25-27$ & 20 & 9 & 4 & 32 & 4 & - & - & - & 2 & 4 & 22 & 3 & 5 & - & 4 & 1 & 100 \\
$1-5,130-132$ & 8 & 3 & 2 & 27 & 1 & - & - & - & 3 & 17 & 17 & 9 & 10 & - & 2 & 3 & 100 \\
$2-1,34-36$ & 6 & 1 & 1 & 52 & 1 & - & - & - & 1 & - & 16 & 6 & 7 & - & 4 & 4 & 100 \\
$2-1,52-53$ & 6 & 1 & 1 & 64 & 1 & - & - & - & 1 & - & 11 & 5 & 6 & - & 2 & 2 & 100 \\
$2-1,128-129$ & 3 & 3 & - & - & 79 & - & - & - & T & - & 4 & 2 & 5 & 2 & 1 & 1 & 100 \\
\hline
\end{tabular}


TABLE 4

Bulk X-Ray Mineralogy, Site 374

\begin{tabular}{|c|c|c|c|c|c|c|c|c|c|c|c|c|c|c|c|c|c|}
\hline $\begin{array}{c}\text { Sample } \\
\text { (Interval in } \mathrm{cm} \text { ) }\end{array}$ & Q & PIF & K F & $\mathrm{C}$ & D & $\mathrm{Ar}$ & G & An & $\mathrm{Ha}$ & Ho & IL & ML & $\mathrm{Sm}$ & At & $\mathrm{Ch}$ & K & Total \\
\hline $\begin{array}{l}1-1,73-75 \\
1-2,50-52\end{array}$ & $\begin{array}{r}9 \\
21\end{array}$ & $\begin{array}{r}2 \\
10\end{array}$ & $\begin{array}{r}3 \\
12\end{array}$ & $\begin{array}{l}36 \\
27\end{array}$ & $\begin{array}{l}5 \\
4\end{array}$ & $\begin{array}{l}9 \\
8\end{array}$ & - & - & $\begin{array}{l}1 \\
1\end{array}$ & $\bar{T}$ & $\begin{array}{r}19 \\
8\end{array}$ & $\begin{array}{l}4 \\
4\end{array}$ & $\begin{array}{l}5 \\
3\end{array}$ & - & $\begin{array}{l}4 \\
1\end{array}$ & $\begin{array}{l}3 \\
1\end{array}$ & $\begin{array}{l}100 \\
100\end{array}$ \\
\hline $\begin{array}{l}2-1,60-62 \\
2-2,60-62 \\
2-3,45-47\end{array}$ & $\begin{array}{l}14 \\
10 \\
14\end{array}$ & $\begin{array}{l}6 \\
3 \\
4\end{array}$ & $\begin{array}{l}1 \\
1 \\
3\end{array}$ & $\begin{array}{l}14 \\
12 \\
14\end{array}$ & $\begin{array}{l}1 \\
T \\
1\end{array}$ & $\begin{array}{l}- \\
-\end{array}$ & $\begin{array}{l}- \\
-\end{array}$ & $\begin{array}{l}- \\
-\end{array}$ & $\begin{array}{l}1 \\
1 \\
1\end{array}$ & $\overline{-}$ & $\begin{array}{l}19 \\
23 \\
15\end{array}$ & $\begin{array}{l}8 \\
7 \\
8\end{array}$ & $\begin{array}{l}17 \\
26 \\
19\end{array}$ & $\begin{array}{l}- \\
-\end{array}$ & $\begin{array}{r}9 \\
8 \\
10\end{array}$ & $\begin{array}{r}10 \\
9 \\
9\end{array}$ & $\begin{array}{l}100 \\
100 \\
100\end{array}$ \\
\hline $3-1,90-92$ & 13 & 3 & 1 & 13 & 2 & $\mathrm{~T}$ & - & - & 2 & - & 23 & 10 & 17 & - & 8 & 8 & 100 \\
\hline $\begin{array}{l}4-2,49-50 \\
4-2,50-51 \\
4-3,50-51 \\
4-4,27-29 \\
4-4,91-92 \\
4-4,92-93\end{array}$ & $\begin{array}{r}18 \\
14 \\
15 \\
10 \\
22 \\
8\end{array}$ & $\begin{array}{r}3 \\
2 \\
2 \\
2 \\
10 \\
2\end{array}$ & $\begin{array}{l}3 \\
1 \\
1 \\
1 \\
4 \\
2\end{array}$ & $\begin{array}{l}10 \\
14 \\
17 \\
26 \\
16 \\
49\end{array}$ & $\begin{array}{l}1 \\
2 \\
3 \\
2 \\
4 \\
3\end{array}$ & $\begin{array}{l}- \\
- \\
- \\
- \\
-\end{array}$ & $\begin{array}{l}- \\
\overline{-} \\
\overline{-} \\
- \\
-\end{array}$ & $\begin{array}{l}- \\
- \\
- \\
- \\
-\end{array}$ & $\begin{array}{l}3 \\
3 \\
3 \\
3 \\
2 \\
5\end{array}$ & $\begin{array}{l}- \\
- \\
- \\
- \\
-\end{array}$ & $\begin{array}{l}20 \\
16 \\
19 \\
25 \\
18 \\
11\end{array}$ & $\begin{array}{l}9 \\
8 \\
9 \\
8 \\
5 \\
6\end{array}$ & $\begin{array}{r}14 \\
18 \\
18 \\
10 \\
8 \\
5\end{array}$ & $\begin{array}{l}- \\
- \\
- \\
- \\
\overline{1}\end{array}$ & $\begin{array}{l}8 \\
8 \\
6 \\
7 \\
6 \\
2\end{array}$ & $\begin{array}{r}11 \\
14 \\
7 \\
6 \\
5 \\
6\end{array}$ & $\begin{array}{l}100 \\
100 \\
100 \\
100 \\
100 \\
100\end{array}$ \\
\hline $\begin{array}{l}5-1,109-111 \\
5-2,5-7 \\
5-2,66-68 \\
5-3,54-56 \\
5-3,100-102 \\
5-4,7-9 \\
5-5,9-11\end{array}$ & $\begin{array}{r}21 \\
8 \\
6 \\
9 \\
4 \\
6 \\
5\end{array}$ & $\begin{array}{l}5 \\
2 \\
2 \\
1 \\
1 \\
1 \\
1 \\
1\end{array}$ & $\begin{array}{l}4 \\
2 \\
2 \\
1 \\
3 \\
2 \\
2 \\
1\end{array}$ & $\begin{array}{l}31 \\
51 \\
59 \\
49 \\
69 \\
56 \\
67\end{array}$ & $\begin{array}{r}10 \\
3 \\
2 \\
2 \\
2 \\
3 \\
3\end{array}$ & $\begin{array}{l}4 \\
- \\
- \\
- \\
- \\
- \\
-\end{array}$ & $\begin{array}{l}- \\
- \\
- \\
- \\
- \\
-\end{array}$ & $\begin{array}{l}- \\
- \\
- \\
- \\
- \\
-\end{array}$ & $\begin{array}{l}3 \\
5 \\
5 \\
6 \\
4 \\
4 \\
5\end{array}$ & $\begin{array}{l}\mathrm{T} \\
- \\
- \\
- \\
- \\
- \\
-\end{array}$ & $\begin{array}{r}13 \\
13 \\
10 \\
11 \\
7 \\
11 \\
7\end{array}$ & $\begin{array}{l}3 \\
5 \\
5 \\
6 \\
5 \\
8 \\
6\end{array}$ & $\begin{array}{l}2 \\
4 \\
3 \\
6 \\
1 \\
2 \\
1\end{array}$ & $\begin{array}{l}1 \\
1 \\
1 \\
\mathrm{~T} \\
\mathrm{~T} \\
1 \\
1\end{array}$ & $\begin{array}{l}1 \\
1 \\
1 \\
1 \\
3 \\
1 \\
2 \\
2 \\
1\end{array}$ & $\begin{array}{l}2 \\
5 \\
4 \\
6 \\
3 \\
5 \\
2\end{array}$ & $\begin{array}{l}100 \\
100 \\
100 \\
100 \\
100 \\
100 \\
100\end{array}$ \\
\hline $\begin{array}{l}6-0,24-26 \\
6-1,64-66 \\
6-2,51-53 \\
6-3,32-34 \\
6-3,68-70 \\
6-4,56-58 \\
6-5,12-14 \\
6-6,65-67\end{array}$ & $\begin{array}{l}9 \\
5 \\
4 \\
4 \\
6 \\
4 \\
6 \\
5\end{array}$ & $\begin{array}{l}2 \\
1 \\
1 \\
2 \\
2 \\
2 \\
1 \\
1 \\
1\end{array}$ & $\begin{array}{l}2 \\
1 \\
2 \\
4 \\
2 \\
2 \\
2 \\
1 \\
1\end{array}$ & $\begin{array}{l}39 \\
54 \\
61 \\
59 \\
59 \\
56 \\
64 \\
53\end{array}$ & $\begin{array}{r}12 \\
4 \\
2 \\
1 \\
1 \\
1 \\
2 \\
1 \\
1 \\
5\end{array}$ & $\begin{array}{l}- \\
- \\
- \\
- \\
- \\
-\end{array}$ & $\begin{array}{l}- \\
- \\
- \\
- \\
- \\
- \\
-\end{array}$ & $\begin{array}{l}- \\
- \\
- \\
- \\
- \\
- \\
-\end{array}$ & $\begin{array}{l}4 \\
5 \\
7 \\
4 \\
8 \\
4 \\
6 \\
4\end{array}$ & $\begin{array}{l}- \\
\overline{-} \\
\overline{-} \\
- \\
-\end{array}$ & $\begin{array}{r}18 \\
11 \\
11 \\
11 \\
10 \\
14 \\
9 \\
13\end{array}$ & $\begin{array}{r}8 \\
10 \\
5 \\
8 \\
6 \\
9 \\
6 \\
7\end{array}$ & $\begin{array}{l}3 \\
3 \\
2 \\
1 \\
1 \\
1 \\
\mathrm{~T} \\
1 \\
3\end{array}$ & $\begin{array}{l}1 \\
\mathrm{~T} \\
1 \\
1 \\
\mathrm{~T} \\
1 \\
1 \\
1\end{array}$ & $\begin{array}{l}2 \\
2 \\
1 \\
2 \\
3 \\
1 \\
1 \\
3\end{array}$ & $\begin{array}{l}5 \\
4 \\
3 \\
3 \\
3 \\
5 \\
3 \\
4\end{array}$ & $\begin{array}{l}100 \\
100 \\
100 \\
100 \\
100 \\
100 \\
100 \\
100\end{array}$ \\
\hline $\begin{array}{l}7-1,83-88 \\
7-2,68-73 \\
7-3,45-47 \\
7-3,72-77 \\
7-4,83-88 \\
7-5,71-76 \\
7-6,52-57\end{array}$ & $\begin{array}{l}5 \\
7 \\
2 \\
6 \\
6 \\
5 \\
6\end{array}$ & $\begin{array}{l}1 \\
2 \\
1 \\
1 \\
1 \\
1 \\
1 \\
1\end{array}$ & $\begin{array}{l}2 \\
1 \\
1 \\
1 \\
1 \\
1 \\
1 \\
1\end{array}$ & $\begin{array}{l}54 \\
60 \\
79 \\
56 \\
52 \\
55 \\
54\end{array}$ & $\begin{array}{l}5 \\
6 \\
2 \\
7 \\
9 \\
7 \\
8\end{array}$ & $\begin{array}{l}- \\
- \\
- \\
- \\
- \\
-\end{array}$ & $\begin{array}{l}- \\
- \\
- \\
- \\
- \\
-\end{array}$ & $\begin{array}{l}- \\
- \\
- \\
- \\
- \\
-\end{array}$ & $\begin{array}{l}3 \\
2 \\
6 \\
2 \\
3 \\
2 \\
3\end{array}$ & $\begin{array}{l}- \\
- \\
- \\
- \\
- \\
-\end{array}$ & $\begin{array}{r}14 \\
10 \\
3 \\
11 \\
11 \\
11 \\
10\end{array}$ & $\begin{array}{r}8 \\
6 \\
3 \\
8 \\
10 \\
9 \\
7\end{array}$ & $\begin{array}{l}2 \\
1 \\
T \\
1 \\
T \\
2 \\
2\end{array}$ & $\begin{array}{l}1 \\
1 \\
1 \\
T \\
1 \\
1 \\
2\end{array}$ & $\begin{array}{l}1 \\
1 \\
1 \\
2 \\
2 \\
2 \\
2 \\
1\end{array}$ & $\begin{array}{l}4 \\
3 \\
1 \\
5 \\
4 \\
4 \\
4 \\
5\end{array}$ & $\begin{array}{l}100 \\
100 \\
100 \\
100 \\
100 \\
100 \\
100\end{array}$ \\
\hline $\begin{array}{l}8-1,83-85 \\
8-2,59-61 \\
8-3,23-25 \\
8-3,46-48 \\
8-4,40-42\end{array}$ & $\begin{array}{l}2 \\
3 \\
6 \\
2 \\
2\end{array}$ & $\begin{array}{l}- \\
1 \\
1 \\
1 \\
\mathrm{~T}\end{array}$ & $\begin{array}{l}1 \\
1 \\
1 \\
1 \\
1\end{array}$ & $\begin{array}{l}79 \\
59 \\
55 \\
70 \\
71\end{array}$ & $\begin{array}{l}3 \\
6 \\
6 \\
4 \\
5\end{array}$ & $\begin{array}{l}- \\
- \\
- \\
-\end{array}$ & $\begin{array}{l}- \\
- \\
- \\
-\end{array}$ & $\begin{array}{l}- \\
- \\
- \\
-\end{array}$ & $\begin{array}{l}6 \\
5 \\
6 \\
4 \\
4\end{array}$ & $\begin{array}{l}- \\
- \\
- \\
-\end{array}$ & $\begin{array}{r}3 \\
10 \\
8 \\
7 \\
6\end{array}$ & $\begin{array}{l}2 \\
6 \\
6 \\
4 \\
4\end{array}$ & $\begin{array}{l}1 \\
1 \\
1 \\
1 \\
1 \\
1\end{array}$ & $\begin{array}{l}\mathrm{T} \\
3 \\
3 \\
2 \\
2\end{array}$ & $\begin{array}{l}1 \\
1 \\
1 \\
1 \\
1\end{array}$ & $\begin{array}{l}2 \\
4 \\
5 \\
3 \\
3\end{array}$ & $\begin{array}{l}100 \\
100 \\
100 \\
100 \\
100\end{array}$ \\
\hline $\begin{array}{l}9-1,60-62 \\
9-2,27-29 \\
9-3,40-42 \\
9-4,16-18\end{array}$ & $\begin{array}{l}4 \\
4 \\
5 \\
5\end{array}$ & $\begin{array}{l}1 \\
1 \\
1 \\
1\end{array}$ & $\begin{array}{l}1 \\
2 \\
2 \\
2\end{array}$ & $\begin{array}{l}61 \\
61 \\
63 \\
59\end{array}$ & $\begin{array}{l}4 \\
5 \\
4 \\
6\end{array}$ & $\begin{array}{l}- \\
- \\
-\end{array}$ & $\begin{array}{l}- \\
- \\
-\end{array}$ & $\begin{array}{l}- \\
- \\
-\end{array}$ & $\begin{array}{l}4 \\
4 \\
5 \\
5\end{array}$ & $\begin{array}{l}- \\
- \\
-\end{array}$ & $\begin{array}{r}9 \\
9 \\
6 \\
10\end{array}$ & $\begin{array}{l}7 \\
5 \\
7 \\
5\end{array}$ & $\begin{array}{l}2 \\
1 \\
1 \\
1\end{array}$ & $\begin{array}{l}3 \\
4 \\
2 \\
2\end{array}$ & $\begin{array}{l}1 \\
1 \\
1 \\
1\end{array}$ & $\begin{array}{l}3 \\
3 \\
3 \\
3\end{array}$ & $\begin{array}{l}100 \\
100 \\
100 \\
100\end{array}$ \\
\hline $10-1,137-139$ & 2 & 1 & 1 & 64 & 7 & - & - & - & 3 & - & 8 & 8 & 1 & 2 & 1 & 2 & 100 \\
\hline $\begin{array}{l}11-1,124-126 \\
11-2,138-141\end{array}$ & $\begin{array}{l}3 \\
5\end{array}$ & $\begin{array}{l}\mathrm{T} \\
\mathrm{T}\end{array}$ & $\begin{array}{l}\mathrm{T} \\
\mathrm{T}\end{array}$ & $\begin{array}{l}21 \\
-\end{array}$ & $\begin{array}{l}55 \\
73\end{array}$ & $\overline{-}$ & - & $\begin{array}{l}- \\
-\end{array}$ & $\begin{array}{l}4 \\
4\end{array}$ & - & $\begin{array}{l}5 \\
6\end{array}$ & $\begin{array}{l}5 \\
7\end{array}$ & $\begin{array}{l}1 \\
1\end{array}$ & $\begin{array}{l}3 \\
1\end{array}$ & $\overline{\mathrm{T}}$ & $\begin{array}{l}3 \\
2\end{array}$ & $\begin{array}{l}100 \\
100\end{array}$ \\
\hline $\begin{array}{l}12-1,80-82 \\
12-2,50-52\end{array}$ & $\begin{array}{l}13 \\
12\end{array}$ & $\begin{array}{l}2 \\
2\end{array}$ & $\begin{array}{l}1 \\
1\end{array}$ & $\overline{-}$ & $\begin{array}{l}20 \\
27\end{array}$ & - & - & - & $\begin{array}{l}6 \\
4\end{array}$ & - & $\begin{array}{l}22 \\
18\end{array}$ & $\begin{array}{l}16 \\
12\end{array}$ & $\begin{array}{r}6 \\
12\end{array}$ & - & $\begin{array}{l}7 \\
6\end{array}$ & $\begin{array}{l}7 \\
6\end{array}$ & $\begin{array}{l}100 \\
100\end{array}$ \\
\hline $\begin{array}{l}13-1,98-100 \\
13-2,60-62 \\
13-3,56-58\end{array}$ & $\begin{array}{l}13 \\
15 \\
14\end{array}$ & $\begin{array}{l}2 \\
2 \\
2\end{array}$ & $\begin{array}{l}1 \\
1 \\
1\end{array}$ & $\begin{array}{l}- \\
- \\
-\end{array}$ & $\begin{array}{l}24 \\
15 \\
17\end{array}$ & $\begin{array}{l}- \\
-\end{array}$ & $\begin{array}{l}- \\
- \\
-\end{array}$ & $\begin{array}{l}- \\
- \\
-\end{array}$ & $\begin{array}{l}4 \\
6 \\
6\end{array}$ & $\begin{array}{l}- \\
-\end{array}$ & $\begin{array}{l}22 \\
20 \\
20\end{array}$ & $\begin{array}{r}9 \\
12 \\
11\end{array}$ & $\begin{array}{l}11 \\
17 \\
14\end{array}$ & $\begin{array}{l}- \\
-\end{array}$ & $\begin{array}{l}7 \\
6 \\
8\end{array}$ & $\begin{array}{l}7 \\
6 \\
7\end{array}$ & $\begin{array}{l}100 \\
100 \\
100\end{array}$ \\
\hline $\begin{array}{l}14-1,50-52 \\
14-2,50-52\end{array}$ & $\begin{array}{l}16 \\
18\end{array}$ & $\begin{array}{l}2 \\
3\end{array}$ & $\begin{array}{l}1 \\
2\end{array}$ & $\overline{-}$ & $\begin{array}{l}15 \\
19\end{array}$ & $\begin{array}{l}- \\
-\end{array}$ & - & $\begin{array}{l}- \\
-\end{array}$ & $\begin{array}{l}6 \\
5\end{array}$ & $\overline{-}$ & $\begin{array}{l}21 \\
18\end{array}$ & $\begin{array}{l}14 \\
10\end{array}$ & $\begin{array}{l}16 \\
14\end{array}$ & - & $\begin{array}{l}5 \\
6\end{array}$ & $\begin{array}{l}4 \\
5\end{array}$ & $\begin{array}{l}100 \\
100\end{array}$ \\
\hline $\begin{array}{l}15-1,50-52 \\
15-2,50-52\end{array}$ & $\begin{array}{l}15 \\
16\end{array}$ & $\begin{array}{l}2 \\
5\end{array}$ & $\begin{array}{l}1 \\
2\end{array}$ & $\overline{-}$ & $\begin{array}{l}12 \\
16\end{array}$ & - & - & - & $\begin{array}{l}5 \\
5\end{array}$ & - & $\begin{array}{l}26 \\
27\end{array}$ & $\begin{array}{r}11 \\
6\end{array}$ & $\begin{array}{l}18 \\
12\end{array}$ & - & $\begin{array}{l}6 \\
8\end{array}$ & $\begin{array}{l}4 \\
3\end{array}$ & $\begin{array}{l}100 \\
100\end{array}$ \\
\hline 25, SWC & 1 & - & - & - & 84 & - & - & - & 7 & - & 2 & 4 & $\mathrm{~T}$ & 1 & - & 1 & 100 \\
\hline 26 , SWC & 4 & $\mathrm{~T}$ & 1 & 70 & 8 & - & $\mathrm{T}$ & - & 3 & - & 4 & 3 & $\mathrm{~T}$ & 2 & 1 & 3 & 100 \\
\hline
\end{tabular}


TABLE 5

Bulk X-Ray Mineralogy, Site 375

\begin{tabular}{|c|c|c|c|c|c|c|c|c|c|c|c|c|c|c|c|c|c|c|}
\hline $\begin{array}{c}\text { Sample } \\
\text { (Interval in } \mathrm{cm} \text { ) }\end{array}$ & Q & PIF & K F & $\mathrm{C}$ & D & Ar & G & An & $\mathrm{Ha}$ & Ho & $\mathrm{Se}$ & IL & ML & $\mathrm{Sm}$ & At & $\mathrm{Ch}$ & $\mathrm{K}$ & Total \\
\hline $1-1,148-150$ & - & - & - & - & - & - & 100 & - & - & - & - & - & - & - & - & - & - & 100 \\
\hline $\begin{array}{l}2-2,37-40 \\
2-2,89-92 \\
2-3,0-3 \\
2-3,39-41 \\
2-3,131-133 \\
2-4,3-5\end{array}$ & $\begin{array}{l}6 \\
- \\
9 \\
5 \\
5 \\
8\end{array}$ & $\begin{array}{c}2 \\
- \\
6 \\
2 \\
2 \\
3\end{array}$ & $\begin{array}{l}\mathrm{T} \\
- \\
2 \\
1 \\
- \\
-\end{array}$ & $\begin{array}{r}53 \\
- \\
18 \\
4 \\
27 \\
36\end{array}$ & $\begin{array}{l}3 \\
- \\
- \\
- \\
9 \\
8\end{array}$ & $\begin{array}{l}- \\
\overline{-} \\
\overline{-}\end{array}$ & $\begin{array}{r}- \\
100 \\
4 \\
62 \\
1 \\
3\end{array}$ & $\begin{array}{l}- \\
- \\
- \\
-\end{array}$ & $\begin{array}{l}\mathrm{T} \\
- \\
- \\
- \\
- \\
\overline{1}\end{array}$ & $\begin{array}{l}\mathrm{T} \\
- \\
- \\
- \\
- \\
-\end{array}$ & $\begin{array}{l}\mathrm{T} \\
\overline{1} \\
- \\
- \\
-\end{array}$ & $\begin{array}{l}3 \\
- \\
8 \\
3 \\
3 \\
9\end{array}$ & $\begin{array}{l}5 \\
- \\
7 \\
5 \\
4 \\
7\end{array}$ & $\begin{array}{l}22 \\
- \\
41 \\
16 \\
44 \\
15\end{array}$ & $\begin{array}{l}4 \\
- \\
- \\
- \\
4\end{array}$ & $\begin{array}{l}1 \\
- \\
3 \\
1 \\
1 \\
4\end{array}$ & $\begin{array}{l}\mathrm{T} \\
- \\
1 \\
1 \\
4 \\
2\end{array}$ & $\begin{array}{l}100 \\
100 \\
100 \\
100 \\
100 \\
100\end{array}$ \\
\hline $\begin{array}{l}4-1,113-115 \\
4-2,65-67 \\
4-3,65-68 \\
4-4,74-77 \\
4-5,76-78 \\
4, C C\end{array}$ & $\begin{array}{r}12 \\
10 \\
8 \\
5 \\
6 \\
8\end{array}$ & $\begin{array}{l}3 \\
3 \\
3 \\
2 \\
3 \\
4\end{array}$ & $\begin{array}{l}\bar{T} \\
1 \\
\mathrm{~T} \\
\overline{1}\end{array}$ & $\begin{array}{l}32 \\
25 \\
45 \\
18 \\
31 \\
29\end{array}$ & $\begin{array}{l}12 \\
15 \\
12 \\
43 \\
12 \\
11\end{array}$ & $\begin{array}{l}- \\
- \\
- \\
-\end{array}$ & $\begin{array}{l}- \\
- \\
- \\
-\end{array}$ & $\begin{array}{l}- \\
\overline{-} \\
- \\
- \\
-\end{array}$ & $\begin{array}{l}- \\
\overline{-} \\
- \\
-\end{array}$ & $\begin{array}{l}- \\
- \\
- \\
- \\
-\end{array}$ & $\begin{array}{l}1 \\
1 \\
1 \\
1 \\
1 \\
T \\
1\end{array}$ & $\begin{array}{r}19 \\
21 \\
8 \\
14 \\
9 \\
9\end{array}$ & $\begin{array}{l}5 \\
5 \\
6 \\
5 \\
9 \\
5\end{array}$ & $\begin{array}{r}9 \\
9 \\
12 \\
7 \\
22 \\
21\end{array}$ & $\begin{array}{l}2 \\
1 \\
- \\
\overline{3} \\
3\end{array}$ & $\begin{array}{l}4 \\
7 \\
2 \\
4 \\
3 \\
5\end{array}$ & $\begin{array}{l}1 \\
3 \\
2 \\
1 \\
2 \\
3\end{array}$ & $\begin{array}{l}100 \\
100 \\
100 \\
100 \\
100 \\
100\end{array}$ \\
\hline $\begin{array}{l}5-1,50-52 \\
5-2,43-45 \\
5-3,40-43 \\
5-4,97-99 \\
5-5,31-32 \\
5-6,98-100\end{array}$ & $\begin{array}{l}8 \\
8 \\
7 \\
7 \\
8 \\
8\end{array}$ & $\begin{array}{r}7 \\
6 \\
3 \\
10 \\
3 \\
4\end{array}$ & $\begin{array}{l}2 \\
2 \\
1 \\
1 \\
1 \\
1\end{array}$ & $\begin{array}{l}26 \\
26 \\
10 \\
17 \\
39 \\
30\end{array}$ & $\begin{array}{r}20 \\
6 \\
1 \\
4 \\
4 \\
4\end{array}$ & $\begin{array}{l}2 \\
- \\
- \\
-\end{array}$ & $\begin{array}{l}- \\
- \\
- \\
- \\
-\end{array}$ & $\begin{array}{l}- \\
\overline{-} \\
- \\
-\end{array}$ & $\begin{array}{l}- \\
\bar{z} \\
\bar{z} \\
\overline{-}\end{array}$ & $\begin{array}{l}- \\
\overline{-} \\
\bar{T} \\
- \\
-\end{array}$ & $\begin{array}{l}1 \\
1 \\
2 \\
2 \\
1 \\
2\end{array}$ & $\begin{array}{r}10 \\
15 \\
13 \\
21 \\
11 \\
9\end{array}$ & $\begin{array}{l}5 \\
6 \\
9 \\
5 \\
8 \\
9\end{array}$ & $\begin{array}{l}13 \\
16 \\
38 \\
18 \\
19 \\
22\end{array}$ & $\begin{array}{l}\overline{4} \\
- \\
- \\
- \\
-\end{array}$ & $\begin{array}{r}4 \\
8 \\
4 \\
13 \\
4 \\
4\end{array}$ & $\begin{array}{l}2 \\
2 \\
5 \\
2 \\
2 \\
4\end{array}$ & $\begin{array}{l}100 \\
100 \\
100 \\
100 \\
100 \\
100\end{array}$ \\
\hline $\begin{array}{l}6-1,58-61 \\
6-3,102-104 \\
6-4,142-145 \\
6-5,52-54 \\
6-6,34-36\end{array}$ & $\begin{array}{r}7 \\
7 \\
11 \\
8 \\
9\end{array}$ & $\begin{array}{l}3 \\
8 \\
8 \\
3 \\
2\end{array}$ & $\begin{array}{l}1 \\
1 \\
1 \\
1 \\
1\end{array}$ & $\begin{array}{l}18 \\
20 \\
23 \\
28 \\
26\end{array}$ & $\begin{array}{l}3 \\
4 \\
4 \\
5 \\
2\end{array}$ & $\begin{array}{l}- \\
\overline{-} \\
\overline{-}\end{array}$ & $\begin{array}{l}- \\
\overline{-} \\
\overline{-}\end{array}$ & $\begin{array}{l}- \\
- \\
- \\
-\end{array}$ & $\begin{array}{l}- \\
\overline{-} \\
-\end{array}$ & $\begin{array}{l}\bar{T} \\
- \\
- \\
-\end{array}$ & $\begin{array}{l}2 \\
\mathrm{~T} \\
2 \\
1 \\
2\end{array}$ & $\begin{array}{r}10 \\
15 \\
13 \\
9 \\
19\end{array}$ & $\begin{array}{r}12 \\
4 \\
6 \\
7 \\
7\end{array}$ & $\begin{array}{l}35 \\
27 \\
24 \\
28 \\
22\end{array}$ & $\begin{array}{l}\overline{1} \\
\overline{-} \\
-\end{array}$ & $\begin{array}{r}6 \\
10 \\
6 \\
5 \\
7\end{array}$ & $\begin{array}{l}3 \\
2 \\
2 \\
5 \\
3\end{array}$ & $\begin{array}{l}100 \\
100 \\
100 \\
100 \\
100\end{array}$ \\
\hline $\begin{array}{l}7-1,133-135 \\
7-2,101-103 \\
7-3,90-92 \\
7-4,62-64 \\
7-5,67-68 \\
7-6,82-84\end{array}$ & $\begin{array}{l}7 \\
8 \\
8 \\
9 \\
7 \\
7\end{array}$ & $\begin{array}{l}4 \\
6 \\
3 \\
4 \\
5 \\
5\end{array}$ & $\begin{array}{c}1 \\
1 \\
1 \\
\mathrm{~T}^{1} \\
1 \\
1\end{array}$ & $\begin{array}{l}26 \\
22 \\
32 \\
28 \\
25 \\
18\end{array}$ & $\begin{array}{l}4 \\
4 \\
5 \\
4 \\
5 \\
5\end{array}$ & $\begin{array}{l}- \\
\bar{T} \\
\bar{T} \\
-\end{array}$ & $\begin{array}{l}- \\
- \\
- \\
- \\
-\end{array}$ & $\begin{array}{l}- \\
- \\
- \\
- \\
-\end{array}$ & $\begin{array}{l}- \\
- \\
- \\
-\end{array}$ & $\begin{array}{l}- \\
\overline{-} \\
\overline{-} \\
\overline{-}\end{array}$ & $\begin{array}{l}2 \\
2 \\
1 \\
1 \\
1 \\
1 \\
1\end{array}$ & $\begin{array}{l}10 \\
15 \\
13 \\
22 \\
14 \\
16\end{array}$ & $\begin{array}{l}7 \\
5 \\
9 \\
6 \\
9 \\
7\end{array}$ & $\begin{array}{l}27 \\
25 \\
20 \\
18 \\
25 \\
29\end{array}$ & $\begin{array}{l}1 \\
- \\
- \\
- \\
- \\
-\end{array}$ & $\begin{array}{r}8 \\
10 \\
5 \\
6 \\
6 \\
9\end{array}$ & $\begin{array}{l}3 \\
2 \\
3 \\
2 \\
2 \\
2\end{array}$ & $\begin{array}{l}100 \\
100 \\
100 \\
100 \\
100 \\
100\end{array}$ \\
\hline $\begin{array}{l}8-1,30-34 \\
8-2,49-51 \\
8-3,49-51 \\
8-4,62-65 \\
8-5,69-72 \\
8-6,36-38\end{array}$ & $\begin{array}{l}9 \\
4 \\
8 \\
6 \\
7 \\
2\end{array}$ & $\begin{array}{l}8 \\
1 \\
8 \\
1 \\
4 \\
-\end{array}$ & $\begin{array}{l}1 \\
- \\
1 \\
\mathrm{~T} \\
\mathrm{~T} \\
-\end{array}$ & $\begin{array}{r}- \\
63 \\
16 \\
53 \\
1 \\
78\end{array}$ & $\begin{array}{c}1 \\
\mathrm{~T} \\
4 \\
2 \\
1 \\
-\end{array}$ & $\begin{array}{l}- \\
- \\
- \\
- \\
-\end{array}$ & $\begin{array}{l}- \\
- \\
- \\
- \\
-\end{array}$ & $\begin{array}{l}- \\
- \\
- \\
- \\
-\end{array}$ & $\begin{array}{l}- \\
\overline{-} \\
\overline{-}\end{array}$ & $\begin{array}{l}- \\
\overline{-} \\
- \\
\overline{-}\end{array}$ & $\begin{array}{l}1 \\
1 \\
1 \\
1 \\
2 \\
-\end{array}$ & $\begin{array}{r}21 \\
8 \\
18 \\
10 \\
17 \\
2\end{array}$ & $\begin{array}{r}9 \\
5 \\
7 \\
5 \\
13 \\
4\end{array}$ & $\begin{array}{l}36 \\
11 \\
24 \\
14 \\
46 \\
12\end{array}$ & $\begin{array}{l}1 \\
2 \\
3 \\
3 \\
- \\
-\end{array}$ & $\begin{array}{r}12 \\
3 \\
8 \\
2 \\
5 \\
1\end{array}$ & $\begin{array}{l}1 \\
2 \\
2 \\
3 \\
4 \\
1\end{array}$ & $\begin{array}{l}100 \\
100 \\
100 \\
100 \\
100 \\
100\end{array}$ \\
\hline $\begin{array}{l}9-1,45-47 \\
9-2,89-91 \\
9-3,94-96 \\
9-4,123-126 \\
9-5,22-24 \\
9-6,80-82\end{array}$ & $\begin{array}{l}4 \\
6 \\
8 \\
7 \\
4 \\
9\end{array}$ & $\begin{array}{l}- \\
5 \\
5 \\
7 \\
T \\
5\end{array}$ & $\begin{array}{l}\overline{\mathrm{T}} \\
1 \\
1 \\
\overline{1} \\
\overline{1}\end{array}$ & $\begin{array}{l}70 \\
23 \\
43 \\
28 \\
69 \\
-\end{array}$ & $\begin{array}{c}1 \\
4 \\
3 \\
3 \\
\mathrm{~T} \\
5\end{array}$ & $\begin{array}{l}- \\
- \\
- \\
- \\
-\end{array}$ & $\begin{array}{l}- \\
- \\
- \\
- \\
-\end{array}$ & $\begin{array}{l}- \\
- \\
- \\
- \\
-\end{array}$ & $\begin{array}{l}- \\
- \\
- \\
-\end{array}$ & $\begin{array}{l}- \\
- \\
- \\
- \\
-\end{array}$ & $\begin{array}{l}\mathrm{T} \\
\mathrm{T} \\
\mathrm{T} \\
\overline{\mathrm{T}} \\
\mathrm{T}\end{array}$ & $\begin{array}{r}2 \\
16 \\
11 \\
10 \\
4 \\
16\end{array}$ & $\begin{array}{r}4 \\
7 \\
5 \\
8 \\
5 \\
11\end{array}$ & $\begin{array}{l}13 \\
22 \\
13 \\
22 \\
11 \\
36\end{array}$ & $\begin{array}{l}2 \\
4 \\
2 \\
2 \\
2 \\
3 \\
3\end{array}$ & $\begin{array}{r}2 \\
10 \\
5 \\
7 \\
2 \\
10\end{array}$ & $\begin{array}{l}2 \\
3 \\
4 \\
5 \\
1 \\
4\end{array}$ & $\begin{array}{l}100 \\
100 \\
100 \\
100 \\
100 \\
100\end{array}$ \\
\hline $\begin{array}{l}10-1,124-126 \\
10-2,39-42 \\
10-2,80-82 \\
10-3,40-42 \\
10-3,80-82\end{array}$ & $\begin{array}{l}3 \\
3 \\
4 \\
3 \\
3\end{array}$ & $\begin{array}{l}1 \\
\bar{T} \\
- \\
-\end{array}$ & $\begin{array}{l}- \\
- \\
- \\
-\end{array}$ & $\begin{array}{l}60 \\
66 \\
64 \\
54 \\
30\end{array}$ & $\begin{array}{c}3 \\
- \\
- \\
\end{array}$ & $\begin{array}{l}- \\
- \\
- \\
-\end{array}$ & $\begin{array}{l}- \\
\overline{-} \\
\overline{-}\end{array}$ & $\begin{array}{l}- \\
- \\
- \\
-\end{array}$ & $\begin{array}{l}- \\
- \\
- \\
-\end{array}$ & $\begin{array}{l}- \\
- \\
- \\
-\end{array}$ & $\begin{array}{l}- \\
- \\
- \\
-\end{array}$ & $\begin{array}{l}5 \\
2 \\
6 \\
4 \\
5\end{array}$ & $\begin{array}{r}7 \\
10 \\
5 \\
3 \\
4\end{array}$ & $\begin{array}{r}15 \\
15 \\
9 \\
27 \\
44\end{array}$ & $\begin{array}{l}3 \\
\overline{5} \\
- \\
-\end{array}$ & $\begin{array}{l}2 \\
1 \\
2 \\
3 \\
3\end{array}$ & $\begin{array}{r}1 \\
3 \\
2 \\
6 \\
11\end{array}$ & $\begin{array}{l}100 \\
100 \\
100 \\
100 \\
100\end{array}$ \\
\hline $\begin{array}{l}11-1,95-97 \\
11-1,104-106 \\
11-2,21-23\end{array}$ & $\begin{array}{l}3 \\
3 \\
3\end{array}$ & $\begin{array}{l}\mathrm{T} \\
\mathrm{T} \\
-\end{array}$ & $\begin{array}{l}- \\
-\end{array}$ & $\begin{array}{l}41 \\
26 \\
96\end{array}$ & $\begin{array}{r}13 \\
19 \\
1\end{array}$ & $\begin{array}{l}- \\
-\end{array}$ & - & $\begin{array}{l}- \\
- \\
-\end{array}$ & $\begin{array}{l}- \\
-\end{array}$ & $\begin{array}{l}- \\
- \\
-\end{array}$ & $\begin{array}{l}- \\
-\end{array}$ & $\begin{array}{r}4 \\
7 \\
-\end{array}$ & $\begin{array}{r}8 \\
8 \\
-\end{array}$ & $\begin{array}{l}20 \\
18 \\
-\end{array}$ & $\begin{array}{r}6 \\
11 \\
-\end{array}$ & $\begin{array}{r}1 \\
4 \\
-\end{array}$ & $\begin{array}{r}4 \\
4 \\
-\end{array}$ & $\begin{array}{l}100 \\
100 \\
100\end{array}$ \\
\hline $13-1,120-121$ & 12 & - & - & 24 & 1 & - & - & - & - & - & - & 7 & 5 & 30 & - & - & 21 & 100 \\
\hline
\end{tabular}


TABLE 6

Bulk X-Ray Mineralogy, Site 376

\begin{tabular}{|c|c|c|c|c|c|c|c|c|c|c|c|c|c|c|c|c|c|c|c|}
\hline $\begin{array}{c}\text { Sample } \\
\text { (Interval in } \mathrm{cm} \text { ) }\end{array}$ & Q & PIF & K F & $\mathrm{C}$ & D & $\mathrm{Ar}$ & G & An & $\mathrm{Ha}$ & Ho & $\mathrm{Se}$ & $\mathrm{Cl}$ & IL & ML & $\mathrm{Sm}$ & At & $\mathrm{Ch}$ & K & Total \\
\hline $1-1,74-76$ & 9 & 2 & - & 36 & 7 & $\mathrm{~T}$ & 1 & - & 4 & 1 & 1 & - & 8 & 5 & 21 & - & 3 & 2 & 100 \\
\hline $1-1,100-102$ & 9 & 3 & $\mathrm{~T}$ & 44 & 3 & - & - & - & 2 & 1 & $\mathrm{~T}$ & - & 9 & 5 & 16 & - & 6 & 3 & 100 \\
\hline $1-2,95-97$ & 9 & 4 & - & 40 & 2 & - & $\mathrm{T}$ & - & 2 & 1 & 1 & - & 7 & 7 & 21 & - & 3 & 3 & 100 \\
\hline $1-3,50-52$ & 9 & 6 & 1 & 42 & 4 & - & - & - & 1 & 1 & $\mathrm{~T}$ & - & 7 & 5 & 15 & - & 6 & 3 & 100 \\
\hline $1-4,50-52$ & 7 & 2 & 2 & 49 & 1 & - & - & - & 1 & $\mathrm{~T}$ & 1 & - & 4 & 5 & 22 & - & 2 & 4 & 100 \\
\hline $1-5,106-108$ & 8 & 6 & - & 36 & 3 & - & - & - & 1 & 1 & 1 & - & 10 & 5 & 20 & - & 6 & 4 & 100 \\
\hline $1-6,144-146$ & 10 & 6 & 3 & 40 & 4 & - & - & - & 1 & 1 & 1 & - & 7 & 4 & 16 & - & 4 & 3 & 100 \\
\hline $2-1,88-90$ & 8 & 4 & 1 & 40 & 4 & - & - & - & 1 & 1 & 1 & - & 9 & 4 & 19 & - & 4 & 4 & 100 \\
\hline $2-2,71-74$ & 8 & 6 & 1 & 37 & 3 & - & - & - & 2 & $\mathrm{~T}$ & 1 & - & 8 & 7 & 21 & - & 3 & 3 & 100 \\
\hline $2-3,108-110$ & 6 & 3 & - & 35 & 1 & - & - & _ & 1 & 1 & 1 & - & 12 & 7 & 25 & - & 6 & 2 & 100 \\
\hline $2-4,57-59$ & 7 & 2 & - & 42 & 3 & - & - & - & 1 & - & 1 & - & 10 & 6 & 21 & - & 3 & 4 & 100 \\
\hline $3-1,146-148$ & 7 & 4 & - & 51 & 1 & - & - & - & 1 & 1 & 1 & - & 6 & 6 & 16 & - & 3 & 3 & 100 \\
\hline $3-2,120-122$ & 6 & 3 & - & 60 & 3 & - & - & - & 1 & 1 & 1 & - & 4 & 3 & 12 & - & 3 & 3 & 100 \\
\hline $3-3,70-72$ & 10 & 3 & - & 52 & 3 & - & - & - & 1 & 1 & 1 & - & 4 & 5 & 15 & - & 2 & 3 & 100 \\
\hline $3-4,45-47$ & 9 & 5 & - & 40 & 3 & - & - & - & 1 & 1 & 1 & - & 8 & 5 & 19 & - & 5 & 3 & 100 \\
\hline $3-5,107-109$ & 9 & 4 & 1 & 36 & 4 & - & - & - & 1 & 1 & 1 & - & 6 & 7 & 24 & - & 3 & 3 & 100 \\
\hline $4-1,88-90$ & 9 & 4 & - & 45 & 3 & - & - & - & 1 & 1 & 1 & - & 6 & 6 & 18 & - & 3 & 3 & 100 \\
\hline $4-2,85-87$ & 8 & 3 & 1 & 56 & 2 & - & - & - & 1 & $\mathrm{~T}$ & $\mathrm{~T}$ & - & 1 & 6 & 15 & - & 2 & 4 & 100 \\
\hline $5-1,100-102$ & 12 & 5 & 1 & 35 & 3 & - & - & - & 1 & 1 & 2 & - & 7 & 6 & 19 & - & 5 & 3 & 100 \\
\hline $5-2,85-87$ & 7 & 3 & 1 & 44 & 4 & - & - & - & 1 & 1 & 1 & - & 9 & 7 & 13 & 1 & 3 & 5 & 100 \\
\hline $5-3,39-41$ & 4 & 3 & 1 & 58 & 4 & - & - & - & 1 & $\mathrm{~T}$ & 1 & - & 8 & 5 & 10 & - & 2 & 3 & 100 \\
\hline $5-3,125-127$ & 4 & 1 & 1 & 63 & 4 & - & - & - & 1 & - & 1 & - & 4 & 6 & 10 & - & 3 & 2 & 100 \\
\hline $5-4,80-82$ & 6 . & 1 & 1 & 55 & 3 & - & - & - & 1 & - & 1 & - & 6 & 8 & 13 & - & 2 & 3 & 100 \\
\hline $5-5,138-140$ & 4 & 1 & 1 & 64 & 4 & - & - & - & 1 & - & - & - & 6 & 7 & 9 & - & 1 & 2 & 100 \\
\hline $6-1,130-132$ & 6 & 6 & - & 37 & 5 & - & - & - & 1 & 1 & 1 & - & 9 & 6 & 20 & 1 & 4 & 3 & 100 \\
\hline $6-2,80-82$ & 6 & 6 & 1 & 42 & 5 & - & - & - & 1 & 1 & - & - & 7 & 5 & 19 & 1 & 4 & 2 & 100 \\
\hline $6-3,112-114$ & 5 & 1 & - & 35 & 2 & - & - & - & 1 & - & - & - & 7 & 11 & 26 & 2 & 3 & 7 & 100 \\
\hline $6-4,44-46$ & 4 & 1 & - & 66 & 6 & - & - & - & 1 & - & - & - & 6 & 7 & 4 & 3 & 1 & 1 & 100 \\
\hline $7-1,100-102$ & 12 & 5 & 4 & 17 & 23 & - & - & - & $\mathrm{T}$ & - & 1 & - & 8 & 7 & 14 & 5 & 3 & 1 & 100 \\
\hline $7-2,53-55$ & 5 & 3 & 1 & 15 & 27 & - & - & - & $\mathrm{T}$ & - & 1 & - & 15 & 10 & 11 & 5 & 5 & 2 & 100 \\
\hline $7-2,85-87$ & 9 & 5 & 2 & 18 & 28 & - & - & - & $\mathrm{T}$ & - & 2 & - & 10 & 5 & 11 & 2 & 7 & 1 & 100 \\
\hline $8-1,93-95$ & 6 & 3 & 1 & 25 & 10 & - & - & - & $\mathrm{T}$ & - & 1 & - & 9 & 16 & 17 & 5 & 3 & 3 & 100 \\
\hline $8-2,46-48$ & 6 & 3 & 1 & 24 & 24 & - & - & - & $\mathrm{T}$ & - & 1 & - & 6 & 9 & 17 & 4 & 3 & 3 & 100 \\
\hline $8-3,125-127$ & 21 & 12 & 10 & 22 & 11 & - & - & - & $\mathrm{T}$ & 2 & 1 & - & 5 & 3 & 8 & 1 & 3 & 1 & 100 \\
\hline $9-1,97-99$ & 9 & 3 & - & 26 & 11 & - & - & - & $\mathrm{T}$ & - & 1 & - & 9 & 10 & 22 & 5 & 3 & 1 & 100 \\
\hline $9-2,60-62$ & 5 & 3 & - & 23 & 6 & - & - & - & $\mathrm{T}$ & - & 1 & 1 & 10 & 8 & 33 & 3 & 4 & 3 & 100 \\
\hline $9-3,57-59$ & 7 & 3 & 1 & 30 & 4 & - & - & - & $\mathrm{T}$ & $\mathrm{T}$ & 1 & 1 & 7 & 6 & 35 & 2 & 2 & 1 & 100 \\
\hline $9-4,25-27$ & 12 & 13 & 5 & 25 & 13 & - & - & - & $\mathrm{T}$ & $\mathrm{T}$ & 1 & - & 10 & 5 & 9 & 3 & 4 & - & 100 \\
\hline $10-1,83-85$ & 16 & 10 & 4 & 31 & 16 & - & - & - & - & $\mathrm{T}$ & 1 & - & 7 & 3 & 6 & 2 & 3 & 1 & 100 \\
\hline $10-2,65-67$ & 6 & 4 & 2 & 20 & 13 & - & - & - & $\mathrm{T}$ & - & 1 & - & 17 & 11 & 19 & 2 & 4 & 1 & 100 \\
\hline $10-3,108-110$ & 9 & 3 & 1 & 35 & 12 & - & - & - & - & - & 1 & - & 10 & 7 & 14 & 3 & 4 & 1 & 100 \\
\hline $11-1,136-138$ & 8 & 3 & 1 & 26 & 8 & - & - & - & $\mathrm{T}$ & - & 1 & - & 15 & 9 & 21 & 3 & 4 & 1 & 100 \\
\hline $11-2,67-69$ & 10 & 7 & 2 & 40 & 11 & - & - & - & $\mathrm{T}$ & - & 1 & - & 7 & 4 & 12 & 2 & 3 & 1 & 100 \\
\hline $11-3,44-46$ & 7 & 4 & 1 & 26 & 12 & - & - & - & $T$ & - & 1 & - & 13 & 10 & 18 & 3 & 3 & 2 & 100 \\
\hline $12-1,95-97$ & 6 & 2 & - & 37 & 1 & - & - & - & - & - & $\mathrm{T}$ & 1 & 4 & 5 & 39 & 2 & 2 & $\mathrm{~T}$ & 100 \\
\hline $12-2,54-56$ & 3 & $\mathrm{~T}$ & - & 60 & $\mathrm{~T}$ & - & - & - & - & - & - & 1 & 3 & 3 & 26 & 1 & 1 & 1 & 100 \\
\hline $12-3,57-59$ & 8 & 3 & $\mathrm{~T}$ & 25 & 1 & - & - & - & - & $\mathrm{T}$ & $\mathrm{T}$ & - & 4 & 7 & 47 & 1 & 2 & 1 & 100 \\
\hline $12-5,67-69$ & 8 & 6 & - & 30 & 2 & - & 2 & - & - & 1 & 1 & 1 & 7 & 3 & 33 & 1 & 4 & 1 & 100 \\
\hline $13-1,135-137$ & 4 & 2 & $\mathrm{~T}$ & 64 & 1 & - & - & - & - & - & - & 1 & 1 & 4 & 21 & - & 1 & 1 & 100 \\
\hline $13-2,73-75$ & 7 & 3 & $\mathrm{~T}$ & 35 & 1 & - & - & - & - & - & - & 2 & 3 & 4 & 43 & - & 2 & $T$ & 100 \\
\hline $13-3,106-108$ & 3 & $\mathrm{~T}$ & - & 36 & $T$ & - & - & - & - & - & - & 2 & 5 & 3 & 51 & - & 1 & $\mathrm{~T}$ & 100 \\
\hline $13-4,98-100$ & 8 & 1 & - & 21 & $\mathrm{~T}$ & - & - & - & - & - & $\mathbf{T}$ & 1 & 5 & 3 & 57 & - & 2 & 1 & 100 \\
\hline $14, \mathrm{CC}$ & 5 & 1 & - & 23 & - & - & - & - & - & - & $\mathrm{T}$ & 1 & 5 & 6 & 57 & - & 2 & $\mathrm{~T}$ & 100 \\
\hline $15-1,108-110$ & 10 & 11 & 7 & 18 & 5 & - & 14 & - & - & 1 & 1 & $\mathrm{~T}$ & 6 & 4 & 20 & 1 & 1 & $\mathrm{~T}$ & 100 \\
\hline $15-2,78-80$ & 8 & 4 & 1 & 26 & 4 & - & - & - & - & - & 1 & - & 17 & 8 & 22 & 2 & 6 & 1 & 100 \\
\hline $15-3,66-69$ & 6 & 3 & 1 & 41 & 3 & - & 3 & - & - & $\mathrm{T}$ & 1 & - & 6 & 4 & 28 & 2 & 2 & 1 & 100 \\
\hline $16-1,68-70$ & 6 & 4 & $\mathrm{~T}$ & 38 & 2 & - & 6 & - & - & 1 & 1 & - & 7 & 4 & 27 & 2 & 2 & 1 & 100 \\
\hline $17-1,117-118$ & 4 & 5 & $\mathrm{~T}$ & 26 & 2 & - & 18 & - & - & - & 1 & - & 11 & 5 & 21 & 2 & 4 & 1 & 100 \\
\hline $18-1,106-108$ & 5 & 2 & - & 32 & 2 & - & 26 & - & - & - & 1 & - & 2 & 4 & 24 & 1 & 1 & $\mathrm{~T}$ & 100 \\
\hline $20-1,106-108$ & - & - & - & - & - & - & 100 & - & - & - & - & - & - & - & - & - & - & - & 100 \\
\hline
\end{tabular}


TABLE 7

Bulk X-Ray Mineralogy, Site 377

\begin{tabular}{lrrrrrrrrrrrrrrrrrr}
\hline $\begin{array}{c}\text { Sample } \\
\text { (Interval in cm) }\end{array}$ & Q & PIF & KF & C & D & Ar & G & An & Ha & Py & Se & IL & ML & Sm & At & Ch & K & Total \\
\hline $1-1,89-91$ & 8 & 2 & 2 & 52 & 2 & - & - & - & 1 & - & - & 14 & 5 & 5 & 1 & 3 & 4 & 100 \\
$1-2,67-69$ & 10 & 5 & 2 & 33 & 2 & - & - & - & 1 & - & - & 24 & 4 & 8 & 1 & 6 & 4 & 100 \\
$2-1,102-105$ & 8 & 2 & 1 & 49 & 1 & - & - & - & - & - & - & 10 & 10 & 12 & - & 3 & 4 & 100 \\
$3-1,141-143$ & 8 & 8 & 4 & 2 & T & - & - & - & - & 4 & - & 9 & T & 45 & 2 & 5 & 13 & 100 \\
$4-1,84-86$ & 17 & 15 & - & 23 & 4 & - & - & - & - & 2 & 4 & 21 & T & 7 & - & 7 & 1 & 100 \\
$4-1,117-118$ & 5 & - & - & 30 & 4 & - & - & - & - & 4 & - & 38 & T & 12 & 2 & 2 & 4 & 100 \\
\hline
\end{tabular}

TABLE 8

Bulk X-Ray Mineralogy, Site 378

\begin{tabular}{|c|c|c|c|c|c|c|c|c|c|c|c|c|c|c|c|c|c|c|}
\hline $\begin{array}{c}\text { Sample } \\
\text { (Interval in } \mathrm{cm} \text { ) }\end{array}$ & Q & PIF & K F & C & D & $\mathrm{Ar}$ & G & An & $\mathrm{Ha}$ & Ho & $\mathrm{Se}$ & IL & ML & $\mathrm{Sm}$ & At & $\mathrm{Ch}$ & K & Total \\
\hline \multicolumn{19}{|l|}{ Hole 378} \\
\hline $1-1,110-112$ & 9 & 4 & 4 & 26 & 4 & - & - & - & 1 & $\mathrm{~T}$ & 1 & 30 & 4 & 9 & - & 6 & 2 & 100 \\
\hline $1-2,40-42$ & 6 & 3 & 1 & 41 & 9 & - & - & - & 1 & - & 1 & 18 & 5 & 9 & - & 5 & 1 & 100 \\
\hline $2-2,50-52$ & 6 & 2 & 1 & 50 & 6 & - & - & - & 1 & - & 1 & 15 & 6 & 9 & - & 3 & 1 & 100 \\
\hline $2-3,70-71$ & 6 & 3 & 4 & 45 & 8 & - & -. & - & 1 & - & 1 & 12 & 6 & 10 & - & 3 & 1 & 100 \\
\hline $2-4,50-52$ & 7 & 1 & - & 45 & 7 & - & - & - & 1 & - & 1 & 17 & 5 & 11 & - & 3 & 2 & 100 \\
\hline $2-5,50-52$ & 7 & 1 & 1 & 51 & 4 & - & - & - & 1 & - & 1 & 15 & 5 & 9 & - & 3 & 2 & 100 \\
\hline $3-1,120-122$ & 7 & 2 & $\mathrm{~T}$ & 42 & 2 & - & - & - & 1 & - & 1 & 22 & 5 & 11 & - & 5 & 1 & 100 \\
\hline $3-2,70-72$ & 7 & 3 & 1 & 43 & 3 & - & - & - & 1 & - & 1 & 21 & 5 & 8 & - & 6 & 1 & 100 \\
\hline $3-3,50-52$ & 6 & 3 & $\mathrm{~T}$ & 42 & 7 & - & - & - & 1 & - & 1 & 22 & 4 & 7 & - & 6 & 1 & 100 \\
\hline $5-1,147-149$ & 6 & 3 & 1 & 45 & 6 & - & - & - & 1 & $T$ & 1 & 19 & 4 & 10 & - & 4 & 1 & 100 \\
\hline $5-2,50-52$ & 7 & 3 & 1 & 43 & 7 & - & - & - & 1 & - & 1 & 17 & 4 & 11 & - & 4 & 1 & 100 \\
\hline $6-2,59-60$ & 8 & 5 & - & 39 & 4 & - & - & - & 1 & - & 1 & 21 & 4 & 12 & - & 4 & 2 & 100 \\
\hline $6-3,60-62$ & 6 & 9 & - & 39 & 2 & - & - & - & $\mathrm{T}$ & - & 1 & 21 & 4 & 12 & - & 5 & 1 & 100 \\
\hline $7-1,50-52$ & 6 & 2 & 1 & 51 & 1 & - & - & - & $\mathrm{T}$ & - & 1 & 16 & 5 & 9 & - & 5 & 1 & 100 \\
\hline $7-2,50-52$ & 4 & 9 & 3 & 47 & 4 & - & - & - & 1 & - & 1 & 16 & 4 & 6 & - & 4 & 1 & 100 \\
\hline $7-3,89-91$ & 10 & 5 & 3 & 29 & 4 & - & - & - & 1 & $\mathrm{~T}$ & 1 & 19 & 3 & 20 & - & 4 & 1 & 100 \\
\hline $7-4,50-52$ & 5 & 7 & 1 & 45 & 2 & - & - & - & 1 & - & 1 & 17 & 2 & 13 & - & 5 & 1 & 100 \\
\hline $7-5,50-52$ & 8 & 3 & 3 & 48 & 3 & - & - & - & 1 & - & 1 & 13 & 2 & 13 & - & 4 & 1 & 100 \\
\hline $8-1,50-52$ & 5 & 3 & 3 & 40 & 5 & - & - & - & 1 & - & 1 & 20 & 4 & 12 & - & 5 & 1 & 100 \\
\hline $8-2,80-82$ & 7 & 2 & $\mathrm{~T}$ & 40 & 5 & - & - & - & 1 & & 1 & 21 & 4 & 13 & - & 5 & 1 & 100 \\
\hline $11-3,60-62$ & 7 & 2 & 1 & 43 & 5 & - & - & - & $\mathrm{T}$ & - & 2 & 16 & 6 & 10 & - & 6 & 2 & 100 \\
\hline $11-4,53-55$ & 8 & 3 & $\mathrm{~T}$ & 45 & 5 & - & - & - & $\mathrm{T}$ & - & 2 & 15 & 7 & 10 & - & 4 & 1 & 100 \\
\hline
\end{tabular}

TABLE 9

Bulk X-Ray Mineralogy, Site 378A

Sample

(Interval in cm) Q PIF KF C D Ar G An Ha Ho Se IL ML Sm At Ch $\mathrm{K}$ Total

\begin{tabular}{|c|c|c|c|c|c|c|c|c|c|c|c|c|c|c|c|c|c|c|}
\hline \multicolumn{19}{|l|}{ Hole 378A } \\
\hline $1-1,120-122$ & 6 & 19 & 1 & 41 & 2 & - & - & - & 1 & - & 1 & 13 & 4 & 9 & - & 3 & 1 & 100 \\
\hline $1-2,70-72$ & 9 & 8 & 1 & 31 & 4 & - & - & - & 1 & $\mathrm{~T}$ & 1 & 23 & 4 & 10 & - & 5 & 2 & 100 \\
\hline $1-3,70-72$ & 7 & 8 & $\mathrm{~T}$ & 46 & 1 & - & - & - & 1 & - & 1 & 15 & 4 & 13 & - & 3 & 1 & 100 \\
\hline $1-4,49-51$ & 8 & 4 & 4 & 37 & 2 & - & - & - & 1 & $\mathrm{~T}$ & 1 & 18 & 4 & 14 & - & 5 & 2 & 100 \\
\hline $1-5,70-72$ & 7 & 2 & 2 & 56 & 1 & - & - & - & 1 & - & 1 & 13 & 4 & 11 & - & 2 & 2 & 100 \\
\hline $3-1,56-58$ & 8 & 4 & - & 47 & 3 & - & $\mathrm{T}$ & - & $\mathrm{T}$ & $\mathrm{T}$ & 3 & 12 & 8 & 9 & - & 4 & 1 & 100 \\
\hline $3-2,73-76$ & 6 & 5 & 3 & 36 & 9 & - & $\mathrm{T}$ & - & $\mathrm{T}$ & 1 & 3 & 16 & 6 & 7 & - & 6 & 1 & 100 \\
\hline $3-3,71-74$ & 7 & 4 & 1 & 44 & 4 & _- & $\mathrm{T}$ & - & $\mathrm{T}$ & $\mathrm{T}$ & 3 & 17 & 7 & 7 & - & 6 & 1 & 100 \\
\hline $3-4,58-61$ & 9 & 4 & 1 & 45 & 3 & - & $\mathrm{T}$ & - & $\mathrm{T}$ & $\mathrm{T}$ & 3 & 14 & 7 & 8 & - & 4 & 2 & 100 \\
\hline $3-5,41-43$ & 8 & 5 & 1 & 43 & 6 & - & $\mathrm{T}$ & - & $\mathrm{T}$ & $\mathrm{T}$ & 3 & 15 & 6 & 7 & - & 5 & 1 & 100 \\
\hline $3-6,71-73$ & 7 & 4 & 1 & 41 & 4 & - & $T$ & - & $\mathrm{T}$ & $\mathrm{T}$ & 5 & 19 & 6 & 7 & - & 5 & 1 & 100 \\
\hline
\end{tabular}


F. MÉLIÈRES

TABLE 10

Clay Mineralogy Data, Site $371^{\mathrm{a}}(<2 \mu \mathrm{m}$ carbonate free fraction)

\begin{tabular}{|c|c|c|c|c|c|c|c|}
\hline $\begin{array}{c}\text { Sample } \\
\text { (Interval in cm) }\end{array}$ & Illite & $\begin{array}{c}\text { Mixed } \\
\text { Layer } \\
10-14 \mathrm{M}\end{array}$ & $\begin{array}{c}\text { Mixed } \\
\text { Layer } \\
10-14 \mathrm{C}\end{array}$ & $\begin{array}{c}\text { Smec- } \\
\text { tite }\end{array}$ & $\begin{array}{l}\text { Atta- } \\
\text { pulgite }\end{array}$ & $\begin{array}{l}\text { Chlo- } \\
\text { rite }\end{array}$ & Kaolinite \\
\hline $1-1,129-131$ & 39 & 24 & - & - & - & 14 & 23 \\
\hline $1-2,48-50$ & 53 & 16 & - & - & _- & 19 & 12 \\
\hline $1-2,113-115$ & 40 & 24 & - & - & - & 15 & 21 \\
\hline $1-3,69-71$ & 42 & 20 & - & - & - & 18 & 20 \\
\hline $1-4,49-51$ & 40 & 25 & - & - & - & 16 & 19 \\
\hline $1-5,44-46$ & 42 & 22 & - & - & - & 19 & 17 \\
\hline $1-5,79-80$ & 43 & 25 & - & - & - & 17 & 15 \\
\hline $1-6,45-47$ & 43 & 25 & - & - & - & 13 & 19 \\
\hline $\begin{array}{l}2-1,127-129 \\
2-2,49-51\end{array}$ & $\begin{array}{l}39 \\
42\end{array}$ & $\begin{array}{l}27 \\
22\end{array}$ & - & - & - & 16 & 18 \\
\hline $2-3,49-51$ & 42 & 25 & - & - & - & 14 & 19 \\
\hline $3-1,79-81$ & 42 & 23 & - & - & - & 17 & 18 \\
\hline $3-2,49-51$ & 38 & 24 & - & - & - & 18 & 20 \\
\hline $3-3,49-51$ & 38 & 26 & - & - & - & 18 & 18 \\
\hline $3-4,47-49$ & 37 & 24 & - & - & - & 13 & 26 \\
\hline $3-5,50-52$ & 39 & 25 & - & - & - & 16 & 20 \\
\hline $3-6,49-51$ & 47 & 28 & - & - & - & 11 & 14 \\
\hline $4-2,49-51$ & 36 & 23 & - & - & - & 20 & 21 \\
\hline $4-3,49-51$ & 35 & 23 & - & - & - & 19 & 23 \\
\hline $4-4,48-50$ & 39 & 20 & - & - & - & 19 & 22 \\
\hline $4-5,49-51$ & 35 & 20 & - & - & - & 17 & 28 \\
\hline $4-6,46-47$ & 41 & 15 & - & - & - & 21 & 23 \\
\hline $4-6,92-94$ & 36 & 20 & - & - & - & 22 & 22 \\
\hline $5-1,36-38$ & 40 & 16 & - & - & - & 18 & 26 \\
\hline $5-1,78-80$ & 36 & 22 & - & - & - & 18 & 24 \\
\hline $5-2,49-51$ & 38 & 15 & - & - & - & 19 & 28 \\
\hline $5-3,49-51$ & 33 & 21 & - & - & - & 19 & 27 \\
\hline $5-4,46-48$ & 46 & 17 & - & - & - & 24 & 13 \\
\hline $5-5,69-71$ & 35 & 18 & - & - & - & 22 & 25 \\
\hline $5-6,50-52$ & 37 & 19 & - & - & - & 19 & 25 \\
\hline $5, \mathrm{CC}$ & 40 & 19 & - & - & - & 17 & 24 \\
\hline $6, \mathrm{CC}$ & 42 & 17 & - & - & - & 17 & 24 \\
\hline $7, \mathrm{CC}$ & 41 & 15 & - & - & - & 21 & 23 \\
\hline $8-2,95-97$ & 34 & 17 & - & - & - & 26 & 23 \\
\hline $8-3,59-61$ & 33 & 15 & - & - & - & 25 & 27 \\
\hline
\end{tabular}

TABLE 11

Clay Mineralogy Data, Site $372^{\mathrm{a}}(<5 \mu \mathrm{m}$, carbonate free fraction)

\begin{tabular}{|c|c|c|c|c|c|c|c|}
\hline $\begin{array}{c}\text { Sample } \\
\text { (Interval in } \mathrm{cm} \text { ) }\end{array}$ & Illite & $\begin{array}{c}\text { Mixed } \\
\text { Layer } \\
10-14 \mathrm{M}\end{array}$ & $\begin{array}{c}\text { Mixed } \\
\text { Layer } \\
14 \mathrm{M}-14 \mathrm{C}\end{array}$ & $\begin{array}{l}\text { Smec- } \\
\text { tite }\end{array}$ & $\begin{array}{l}\text { Atta- } \\
\text { pulgite }\end{array}$ & $\begin{array}{l}\text { Chlo- } \\
\text { rite }\end{array}$ & Kaolinite \\
\hline $1-1,128-130$ & 65 & 10 & - & - & - & 20 & 5 \\
\hline $1-2,53-55$ & 65 & 10 & - & - & - & 20 & 5 \\
\hline $1 \cdot 3,49-51$ & 65 & 10 & - & - & - & 20 & 5 \\
\hline $1-4,40-41$ & 60 & 20 & - & - & - & 10 & 10 \\
\hline $2-1,129-132$ & 65 & 15 & - & - & - & 12 & 8 \\
\hline $2-2,45-47$ & 60 & 10 & - & - & - & 20 & 10 \\
\hline $2-3,50-52$ & 60 & 20 & - & - & - & 12 & 8 \\
\hline $2-4,11-13$ & 65 & 15 & - & - & - & 12 & 8 \\
\hline $2-4,64-66$ & 60 & 15 & - & - & - & 15 & 10 \\
\hline $3-1,70-72$ & 70 & 10 & - & - & - & 12 & 8 \\
\hline $3-2,50-52$ & 60 & 15 & - & - & - & 15 & 10 \\
\hline $3-3,50-52$ & 55 & 25 & - & - & - & 15 & 5 \\
\hline $4-1,103-105$ & 35 & - & - & 50 & - & 15 & - \\
\hline $4-2,50-52$ & 30 & - & - & 50 & - & 20 & - \\
\hline $5-1,137-139$ & 36 & - & - & 40 & - & 20 & 4 \\
\hline $6-1,145-147$ & 30 & - & - & 45 & - & 20 & 5 \\
\hline $9-1,127-129$ & 32 & - & - & 50 & - & 18 & - \\
\hline $9-2,69-71$ & 40 & - & - & 40 & - & 20 & - \\
\hline $9-3,60-62$ & 30 & - & - & 60 & - & 10 & - \\
\hline $9-4,50-52$ & 30 & - & - & 50 & - & 20 & - \\
\hline $9, \mathrm{CC}$ & 38 & - & - & 37 & - & 25 & - \\
\hline $10-1,100-102$ & 40 & - & - & 40 & - & 20 & - \\
\hline $10-2,80-82$ & 40 & - & - & 40 & - & 20 & - \\
\hline $11-1,100-102$ & 50 & - & - & 20 & - & 30 & - \\
\hline $11-2,50-52$ & 45 & - & - & 30 & - & 25 & - \\
\hline $11-3,50-52$ & 45 & - & - & 30 & - & 25 & - \\
\hline $12-1,20-22$ & 45 & 25 & - & - & - & 30 & - \\
\hline $12-3,50-52$ & 50 & 20 & - & - & - & 30 & - \\
\hline $12-4,50-52$ & 48 & 20 & - & - & - & 32 & - \\
\hline $12-5,60-62$ & 48 & 24 & - & - & - & 28 & - \\
\hline $12-6,50-52$ & 45 & 30 & - & - & - & 20 & 5 \\
\hline $13-1,50-52$ & 40 & 35 & - & - & - & 20 & 5 \\
\hline $13-2,50-52$ & 40 & 20 & - & 20 & - & 18 & 2 \\
\hline $13-3,50-52$ & 40 & 20 & - & 10 & - & 25 & 5 \\
\hline $13-4,50-52$ & 40 & 16 & - & 20 & - & 20 & 4 \\
\hline $13-5,50-52$ & 40 & 10 & - & 30 & - & 18 & 2 \\
\hline $13-6,50-52$ & 45 & 5 & - & 30 & - & 18 & 4 \\
\hline $14-1,50-52$ & 40 & - & - & 30 & - & 25 & 5 \\
\hline $14-2,50-52$ & 36 & - & - & 48 & - & 14 & 2 \\
\hline $14-3,50-52$ & 30 & - & - & 45 & - & 20 & 5 \\
\hline $14-4,50-52$ & 40 & - & - & 35 & - & 22 & 3 \\
\hline $14-5,50-52$ & 50 & - & - & 20 & - & 25 & 5 \\
\hline $14-6,60-62$ & 45 & - & - & 30 & - & 23 & 2 \\
\hline $15-1,30-32$ & 45 & 10 & - & 20 & - & 20 & 5 \\
\hline $15-2,50-52$ & 45 & - & - & 30 & - & 25 & - \\
\hline $15-3,30-32$ & 40 & - & - & 32 & - & 28 & - \\
\hline $15-4,50-52$ & 40 & - & - & 35 & - & 25 & - \\
\hline $15-5,60-62$ & 38 & - & - & 40 & - & 22 & - \\
\hline $15-6,50-52$ & 45 & - & - & 35 & - & 20 & - \\
\hline $16-1,60-62$ & 42 & - & - & 36 & - & 22 & - \\
\hline $16-2,50-52$ & 50 & - & - & 42 & - & 8 & - \\
\hline $16-3,50-52$ & 48 & - & - & 44 & - & 8 & - \\
\hline $16-4,50-52$ & 50 & - & - & 44 & - & 6 & - \\
\hline $16-5,60-62$ & 65 & - & - & 30 & - & 5 & - \\
\hline $16-6,48-50$ & 65 & - & - & 30 & - & 5 & - \\
\hline $17-1,78-80$ & 60 & - & - & 30 & - & 10 & - \\
\hline $17-2,50-52$ & 50 & - & - & 45 & - & 5 & - \\
\hline $17-3,50-52$ & 55 & - & - & 40 & - & 5 & - \\
\hline $17-4,50-52$ & 50 & - & - & 35 & - & 15 & - \\
\hline $17-5,60-62$ & 60 & - & - & 30 & - & 10 & - \\
\hline $17-6,50-52$ & 45 & - & - & 35 & - & 20 & - \\
\hline $18-1,80-82$ & 52 & - & - & 24 & - & 24 & - \\
\hline $18-2,50-52$ & 52 & - & - & 24 & - & 24 & - \\
\hline $18-3,50-52$ & 50 & - & - & 26 & - & 24 & - \\
\hline $18-4,50-52$ & 50 & - & - & 30 & - & 20 & - \\
\hline $18-5,50-52$ & 46 & - & - & 36 & - & 18 & - \\
\hline $18-6,50-52$ & 50 & - & - & 30 & - & 20 & - \\
\hline $19-1.50-52$ & 50 & - & - & 32 & - & 18 & - \\
\hline $19-2,50-52$ & 50 & - & - & 30 & - & 20 & - \\
\hline $19-3,50-52$ & 50 & - & - & 30 & - & 20 & - \\
\hline $19-4,50-52$ & 40 & - & - & 42 & - & 18 & - \\
\hline $19-5,50-52$ & 48 & - & - & 30 & - & 22 & - \\
\hline $19-6,50-52$ & 46 & - & - & 38 & - & 16 & - \\
\hline
\end{tabular}


BULK X-RAY MINERALOGY DATA

TABLE 11 - Continued

\begin{tabular}{|c|c|c|c|c|c|c|c|}
\hline $\begin{array}{c}\text { Sample } \\
\text { (Interval in } \mathrm{cm} \text { ) }\end{array}$ & Illite & $\begin{array}{c}\text { Mixed } \\
\text { Layer } \\
10-14 \mathrm{M}\end{array}$ & $\begin{array}{c}\text { Mixed } \\
\text { Layer } \\
14 \mathrm{M}-14 \mathrm{C}\end{array}$ & $\begin{array}{l}\text { Smec- } \\
\text { tite }\end{array}$ & $\begin{array}{l}\text { Atta- } \\
\text { pulgite }\end{array}$ & $\begin{array}{l}\text { Chlo- } \\
\text { rite }\end{array}$ & Kaolinite \\
\hline $20-1,50-52$ & 40 & - & - & 45 & - & 15 & - \\
\hline $20-2,50-52$ & so & - & - & 38 & - & 12 & - \\
\hline $20-3,50-52$ & 50 & - & - & 38 & - & 12 & - \\
\hline $20-4,50-52$ & 50 & - & - & 40 & - & 10 & - \\
\hline $20-5,50-52$ & 55 & - & - & 30 & - & 15 & - \\
\hline $20-6,50-52$ & 65 & - & - & 20 & - & 15 & - \\
\hline $21-2,50-52$ & 75 & - & - & 10 & - & 15 & - \\
\hline $21-3,48-50$ & 70 & - & - & 20 & - & 10 & - \\
\hline $21-4,60-62$ & 75 & - & - & 20 & - & 5 & - \\
\hline $21-5,60-62$ & 65 & - & - & 10 & - & 15 & - \\
\hline $21-6,50-52$ & 65 & - & - & 20 & - & 15 & - \\
\hline $22-1,50-52$ & 55 & - & - & 30 & - & 15 & - \\
\hline $22-2,50-52$ & 55 & - & - & 25 & - & 20 & - \\
\hline $22-3,50-52$ & 45 & - & - & 30 & - & 25 & - \\
\hline $22-4,50-52$ & 45 & - & - & 35 & - & 20 & - \\
\hline $23-1,121-123$ & 35 & - & - & 40 & - & 25 & - \\
\hline $23-2,50-52$ & 40 & - & - & 35 & - & 25 & - \\
\hline $23-3,50-52$ & 40 & - & - & 40 & - & 20 & - \\
\hline $23-4,60-62$ & 45 & - & - & 35 & - & 20 & - \\
\hline $23-5,50-52$ & 40 & - & - & 36 & - & 24 & - \\
\hline $23-6,50-52$ & 40 & - & - & 40 & - & 20 & - \\
\hline $24-1,50-52$ & 40 & - & - & 40 & - & 20 & - \\
\hline $24-3,90-92$ & 40 & - & - & 40 & - & 20 & - \\
\hline $24-4,50-52$ & 40 & - & - & 40 & - & 20 & - \\
\hline $24-5,50-52$ & 40 & - & - & 40 & - & 20 & - \\
\hline $24-6,50-52$ & 45 & 10 & - & 30 & - & 15 & - \\
\hline $25-1,50-52$ & 45 & 10 & - & 30 & - & 15 & - \\
\hline $25-2,50-52$ & 45 & 5 & - & 35 & - & 15 & - \\
\hline $25-3,50-52$ & 50 & 10 & - & 30 & - & 10 & - \\
\hline $25-4,50-52$ & 45 & 10 & - & 30 & - & 15 & - \\
\hline $25-5,50-52$ & 45 & 15 & - & 25 & - & 15 & - \\
\hline $26-1,50-52$ & 50 & 20 & - & 15 & - & 15 & - \\
\hline $26-2,50-52$ & 50 & 25 & - & 10 & - & 15 & - \\
\hline $26-3,50-52$ & 45 & 30 & - & 15 & - & 10 & - \\
\hline $26-4,50-52$ & 40 & 10 & - & 40 & - & 10 & - \\
\hline $26-5,50-52$ & 45 & 10 & - & 35 & - & 10 & - \\
\hline $27-1,40-42$ & 40 & - & - & 50 & - & 10 & - \\
\hline $27-2,50-52$ & 50 & - & - & 35 & - & 15 & - \\
\hline $27-3,50-52$ & 45 & - & - & 40 & - & 15 & - \\
\hline $27-4,50-52$ & 55 & - & - & 35 & - & 10 & - \\
\hline $27-5,50-52$ & 50 & - & - & 35 & - & 15 & - \\
\hline $28-1,100-102$ & 45 & 10 & - & 35 & - & 10 & - \\
\hline $28-2,50-52$ & 40 & - & - & 50 & - & 10 & - \\
\hline $28-3,50-52$ & 35 & - & - & 55 & - & 10 & - \\
\hline $28-4,50-52$ & 35 & - & - & 55 & - & 10 & - \\
\hline $28-5,60-62$ & 35 & - & - & 55 & - & 10 & - \\
\hline $28-6,50-52$ & 40 & - & - & 50 & - & 10 & - \\
\hline $29-1,80-82$ & 40 & - & - & 50 & - & 10 & - \\
\hline $29-2,50-52$ & 45 & - & - & 45 & - & 10 & - \\
\hline $29-3,50-52$ & 45 & - & - & 40 & - & 15 & - \\
\hline $29-4,50-52$ & 45 & $\bar{a}$ & - & 40 & - & 15 & - \\
\hline $29-5,50-52$ & 40 & 25 & - & 20 & - & 15 & - \\
\hline $30-2,50-52$ & 40 & 30 & - & 20 & - & 10 & - \\
\hline $30-3,50-52$ & 40 & 25 & - & 20 & - & 15 & - \\
\hline $30-4,50-52$ & 40 & 25 & - & 20 & - & 15 & - \\
\hline $30-5,50-52$ & 40 & 35 & - & 10 & - & 15 & - \\
\hline $30-6,51-53$ & 45 & 20 & - & 25 & - & 10 & - \\
\hline $31-1,105-107$ & 45 & 20 & - & 25 & - & 10 & - \\
\hline $31-2,50-52$ & 50 & 15 & - & 25 & - & 10 & - \\
\hline $31-3,43-44$ & so & 20 & - & 20 & - & 10 & - \\
\hline $31-4,26-28$ & 45 & 25 & - & 20 & - & 10 & - \\
\hline $31-5,50-52$ & so & 45 & - & - & - & 10 & - \\
\hline $31-6,29-31$ & 50 & 45 & - & - & - & 5 & - \\
\hline $32-1,50-52$ & 50 & 30 & - & 10 & - & 10 & - \\
\hline $32-3,70-72$ & 55 & 30 & - & 10 & - & 5 & - \\
\hline $32-4,57-59$ & 40 & 40 & - & 10 & - & 10 & - \\
\hline $32-5,47-49$ & 40 & 40 & - & 10 & - & 10 & - \\
\hline $32-6,60-63$ & 60 & 30 & - & - & - & 10 & - \\
\hline $33-1,37-39$ & 65 & 25 & - & - & - & 10 & - \\
\hline $33-2,50-52$ & 70 & 20 & - & - & - & 10 & - \\
\hline $33-3,40-42$ & 75 & 20 & - & - & - & 5 & - \\
\hline $33-4,75-77$ & 55 & 10 & - & 10 & - & 25 & - \\
\hline $33-5,60-62$ & 60 & 20 & - & - & - & 20 & - \\
\hline $33-6,71-72$ & 60 & 10 & - & - & - & 30 & - \\
\hline
\end{tabular}

TABLE 11 - Continued

\begin{tabular}{|c|c|c|c|c|c|c|c|}
\hline $\begin{array}{c}\text { Sample } \\
\text { (Interval in } \mathrm{cm} \text { ) }\end{array}$ & Illite & $\begin{array}{c}\text { Mixed } \\
\text { Layer } \\
10-14 \mathrm{M}\end{array}$ & $\begin{array}{c}\text { Mixed } \\
\text { Layer } \\
14 \mathrm{M}-14 \mathrm{C}\end{array}$ & $\begin{array}{l}\text { Smec- } \\
\text { tite }\end{array}$ & $\begin{array}{l}\text { Atta- } \\
\text { pulgite }\end{array}$ & $\begin{array}{l}\text { Chlo- } \\
\text { rite }\end{array}$ & Kaolinite \\
\hline $34-1,127-129$ & 65 & 5 & - & - & - & 30 & - \\
\hline $34-2,42-44$ & 65 & 5 & - & - & - & 30 & - \\
\hline $34-3,69-71$ & 60 & 10 & - & - & - & 30 & - \\
\hline $34-4,28-30$ & 60 & 10 & - & - & - & 30 & - \\
\hline $34-5,15-17$ & 55 & 15 & - & - & - & 30 & - \\
\hline $35-1,102-105$ & $\begin{array}{l}70 \\
65\end{array}$ & $\begin{array}{r}10 \\
5\end{array}$ & - & $\overline{-}$ & $\overline{-}$ & $\begin{array}{l}20 \\
30\end{array}$ & - \\
\hline $\begin{array}{l}35-2,30-32 \\
35-3,67-69\end{array}$ & $\begin{array}{l}65 \\
55\end{array}$ & $\begin{array}{l}5 \\
5\end{array}$ & $\overline{-}$ & $\overline{-}$ & $\overline{-}$ & $\begin{array}{l}30 \\
40\end{array}$ & $\overline{-}$ \\
\hline $36-1,30-32$ & 60 & 5 & - & - & - & 35 & - \\
\hline $36-2,31-33$ & 60 & 5 & - & - & - & 35 & - \\
\hline $36-3,50-52$ & 60 & 5 & - & - & - & 35 & - \\
\hline $36-4,55-58$ & 55 & 5 & - & - & - & 40 & - \\
\hline $36-5,31-33$ & 60 & 5 & - & - & - & 35 & - \\
\hline $36-6,41-43$ & 55 & 10 & - & - & - & 35 & - \\
\hline $37-1,132-134$ & 60 & 10 & - & - & - & 30 & - \\
\hline $37-2,41-43$ & 60 & 10 & - & - & - & 30 & - \\
\hline $37-3,74-76$ & 55 & 5 & - & - & - & 40 & - \\
\hline $37-4,58-60$ & 60 & 10 & - & - & - & 30 & - \\
\hline $38-1,44-46$ & 70 & 5 & - & - & - & 25 & - \\
\hline $38-2,40-42$ & 55 & 15 & - & - & - & 30 & - \\
\hline $38-3,50-52$ & 70 & 10 & - & - & - & 20 & - \\
\hline $38-4,48-50$ & 60 & 10 & - & - & - & 30 & - \\
\hline $38-5,55-57$ & 60 & 10 & - & - & - & 30 & - \\
\hline $38-6,118-120$ & so & - & - & 25 & - & 25 & - \\
\hline $39-1,36-38$ & 70 & 5 & - & - & - & 25 & - \\
\hline $39-2,36-38$ & 70 & 5 & - & - & - & 25 & - \\
\hline $39-3,58-62$ & 70 & 10 & - & - & - & 20 & - \\
\hline $39-4,96-99$ & 75 & 10 & - & - & - & 15 & - \\
\hline $39-5,62-64$ & 80 & 10 & - & - & - & 10 & - \\
\hline $39-6,54-56$ & 70 & 10 & - & - & - & 20 & - \\
\hline $40-1,120-122$ & 70 & 10 & - & - & - & 20 & - \\
\hline $40-2,11-13$ & 75 & 10 & - & - & - & 15 & - \\
\hline $40-3,54-56$ & 70 & 10 & - & - & - & 20 & - \\
\hline $40-4,90-92$ & 70 & 10 & - & - & - & 20 & - \\
\hline $40-5,103-104$ & 55 & - & - & 20 & - & 25 & - \\
\hline $40-6,69-71$ & 55 & - & - & 20 & - & 25 & - \\
\hline $41-1,64-65$ & 50 & 20 & - & - & - & 30 & - \\
\hline $41-2,60-62$ & 55 & 10 & - & - & - & 35 & - \\
\hline $41-3,79-81$ & 65 & 5 & - & - & - & 30 & - \\
\hline $41-4,56-58$ & 60 & 5 & - & 5 & - & 30 & - \\
\hline $41-5,72-74$ & 60 & 10 & - & 5 & - & 25 & - \\
\hline $41-6,64-66$ & 70 & 10 & - & - & - & 20 & - \\
\hline $42-1,60-62$ & 60 & 10 & - & - & - & 30 & - \\
\hline $42-2,67-69$ & 80 & 10 & - & - & - & 10 & - \\
\hline $42-3,68-70$ & 65 & 10 & - & - & - & 25 & - \\
\hline $42-4,47-49$ & 70 & 10 & - & - & - & 20 & - \\
\hline $42-5,61-64$ & 65 & 10 & - & - & - & 25 & - \\
\hline $42-6,52-54$ & 65 & 10 & - & 5 & - & 20 & - \\
\hline $43-2,71-73$ & 65 & 10 & - & 5 & - & 20 & - \\
\hline $43-3,62-64$ & 60 & 10 & - & 10 & - & 20 & - \\
\hline $43-4,99-101$ & 60 & 5 & - & 15 & - & 20 & - \\
\hline $43-5,63-66$ & 70 & - & - & 20 & - & 10 & - \\
\hline $43-6,103-104$ & 60 & 10 & - & 10 & - & 20 & - \\
\hline $44-1,36-38$ & 60 & 10 & - & 10 & - & 20 & - \\
\hline $44-2,30-32$ & 60 & 5 & - & 15 & - & 20 & - \\
\hline $44-3,97-99$ & 55 & 5 & - & 15 & - & 25 & - \\
\hline $44-4,50-53$ & 65 & - & - & 20 & - & 25 & - \\
\hline $44-5,84-86$ & so & - & - & 20 & - & 30 & - \\
\hline $44-6,61-63$ & 50 & - & - & 20 & - & 30 & - \\
\hline $45-1,86-88$ & 60 & - & - & 10 & - & 30 & - \\
\hline $45-2,28-30$ & 70 & - & - & 5 & - & 25 & - \\
\hline $45-3,46-48$ & 70 & - & - & 10 & - & 20 & - \\
\hline $45-4,76-78$ & 70 & - & - & 10 & - & 20 & - \\
\hline $45-5,75-77$ & 75 & - & - & 10 & - & 15 & - \\
\hline $45-6,84-86$ & 70 & - & - & 15 & - & 15 & - \\
\hline $46-3,63-65$ & 65 & - & - & 20 & - & 15 & - \\
\hline
\end{tabular}


TABLE 12

Clay Mineralogy Data, Site $373 \mathrm{~A}^{\mathrm{a}}(<2 \mu \mathrm{m}$, carbonate free fraction)

\begin{tabular}{lccccccc}
\hline $\begin{array}{c}\text { Sample } \\
\text { (Interval in cm) }\end{array}$ & Illite & $\begin{array}{c}\text { Mixed } \\
\text { Layer } \\
10-14 \mathrm{M}\end{array}$ & $\begin{array}{c}\text { Mixed } \\
\text { Layer } \\
10-14 \mathrm{C}\end{array}$ & $\begin{array}{c}\text { Smec- } \\
\text { tite }\end{array}$ & $\begin{array}{c}\text { Atta- } \\
\text { pulgite }\end{array}$ & $\begin{array}{c}\text { Chlo- } \\
\text { rite }\end{array}$ & Kaolinite \\
\hline $1-1,48-50$ & 41 & - & - & - & - & 21 & 38 \\
$1-1,6-66$ & 35 & 20 & - & - & - & 16 & 29 \\
$1-1,88-90$ & 41 & 25 & - & - & - & 14 & 20 \\
$1-2,8-10$ & 27 & 27 & - & - & - & 20 & 26 \\
$1-2,143-145$ & 35 & 19 & - & - & - & 16 & 30 \\
$1-3,51-53$ & 47 & 21 & - & - & - & 12 & 20 \\
$1-5,130-132$ & 39 & 26 & - & - & - & 14 & 21 \\
$2-1,34-36$ & 39 & 12 & - & - & - & 18 & 31 \\
$2-1,52-53$ & 40 & 19 & - & - & - & 17 & 24 \\
$2-1,128-129$ & 35 & - & - & 20 & - & 15 & 30
\end{tabular}

aP. Rouge CFP Lab, Bordeaux, France.

TABLE 13

Clay Mineralogy Data, Site $374^{\mathrm{a}}(<2 \mu \mathrm{m}$, carbonate free fraction)

\begin{tabular}{|c|c|c|c|c|c|c|c|}
\hline $\begin{array}{c}\text { Sample } \\
\text { (Interval in } \mathrm{cm} \text { ) }\end{array}$ & Illite & $\begin{array}{c}\text { Mixed } \\
\text { Layer } \\
10-14 \mathrm{M}\end{array}$ & $\begin{array}{c}\text { Mixed } \\
\text { Layer } \\
14 \mathrm{C}-14 \mathrm{M}\end{array}$ & $\begin{array}{c}\text { Smec- } \\
\text { tite }\end{array}$ & $\begin{array}{l}\text { Atta- } \\
\text { pulgite }\end{array}$ & $\begin{array}{l}\text { Chlo- } \\
\text { rite }\end{array}$ & Kaolinite \\
\hline $1-1,73-75$ & 20 & $T$ & $\mathrm{~T}$ & 55 & 5 & 10 & 10 \\
\hline & & $\mathrm{T}$ & $\mathrm{T}$ & & 5 & 10 & 10 \\
\hline $2-1,60-62$ & 15 & - & $\bar{T}$ & 65 & $\mathrm{~T}$ & T & 20 \\
\hline $\begin{array}{l}2-2,60-62 \\
2-3,45-47\end{array}$ & $\begin{array}{l}10 \\
10\end{array}$ & - & $\begin{array}{l}\mathrm{T} \\
\mathrm{T}\end{array}$ & $\begin{array}{l}65 \\
65\end{array}$ & $\stackrel{?}{\mathrm{~T}}$ & $\begin{array}{l}5 \\
5\end{array}$ & $\begin{array}{l}20 \\
20\end{array}$ \\
\hline $3-1,90-92$ & 15 & - & $\mathrm{T}$ & 65 & - & $\mathrm{T}$ & 20 \\
\hline $4-2,49-50$ & 10 & - & $\mathrm{T}$ & 70 & $?$ & $\mathrm{~T}$ & 20 \\
\hline $4-2,50-51$ & 15 & - & - & 60 & $?$ & $\mathrm{~T}$ & 25 \\
\hline $4-3,50-52$ & 15 & - & $\mathrm{T}$ & 65 & ? & $\mathrm{T}$ & 20 \\
\hline $4-4,27-29$ & 20 & - & - & 55 & - & 10 & 15 \\
\hline $4-4,91-92$ & 15 & - & $\mathrm{T}$ & 60 & $\mathrm{~T}$ & 5 & 20 \\
\hline $4-4,92-93$ & 15 & - & $\mathrm{T}$ & 50 & 15 & 5 & 15 \\
\hline $5-1,109-111$ & 15 & - & $\mathrm{T}$ & 40 & 25 & 5 & 15 \\
\hline $5-2,5-7$ & is & - & $\mathrm{T}$ & 30 & 35 & $\mathrm{~T}$ & 20 \\
\hline $5-2,66-68$ & 15 & - & $\mathrm{T}$ & 25 & 35 & 5 & 20 \\
\hline $5-3,54-56$ & 20 & - & $\mathrm{T}$ & 35 & 25 & 5 & 15 \\
\hline $5-3,100-102$ & 15 & - & $\mathrm{T}$ & 35 & 35 & $\mathrm{~T}$ & 15 \\
\hline $5-4,7-9$ & 20 & - & $\mathrm{T}$ & 35 & 25 & 5 & 15 \\
\hline $5-5,9-11$ & 20 & - & $\mathrm{T}$ & 35 & 25 & 5 & 15 \\
\hline $6-0,24-26$ & 25 & - & $\mathrm{T}$ & 35 & 15 & 10 & 15 \\
\hline $6-1,64-66$ & 25 & $\mathrm{~T}$ & - & 35 & 15 & 10 & 15 \\
\hline $6-2,51-53$ & 30 & $\mathrm{~T}$ & $\mathrm{~T}$ & 40 & 10 & 10 & 10 \\
\hline $6-3,32-34$ & 15 & - & $\mathrm{T}$ & 30 & 35 & 5 & 15 \\
\hline $6-3,68-70$ & 25 & - & $\mathrm{T}$ & 35 & 15 & 10 & 15 \\
\hline $6-4,56-58$ & 25 & - & - & 20 & 35 & 5 & 15 \\
\hline $6-5,12-14$ & 15 & - & $\mathrm{T}$ & 20 & 45 & 5 & 15 \\
\hline $6-6,65-67$ & 25 & $\mathrm{~T}$ & $\mathrm{~T}$ & 45 & 10 & 10 & 10 \\
\hline $7-1,83-88$ & 20 & - & $\mathrm{T}$ & 25 & 35 & 5 & 15 \\
\hline $7-2,68-73$ & 25 & - & $\mathrm{T}$ & 25 & 30 & 10 & 10 \\
\hline $7-3,45-47$ & 30 & - & $\mathrm{T}$ & 30 & 20 & 10 & 10 \\
\hline $7-3,72-77$ & 30 & - & - & 30 & 15 & 10 & 15 \\
\hline $7-4,83-88$ & 20 & - & $\mathrm{T}$ & 25 & 30 & 5 & 20 \\
\hline $7-5,71-76$ & 20 & - & $\mathrm{T}$ & 30 & 25 & 10 & 15 \\
\hline $7-6,52-57$ & 15 & - & - & 25 & 45 & 5 & 10 \\
\hline $8-1,83-85$ & 20 & - & $\mathrm{T}$ & 35 & 20 & 10 & 15 \\
\hline $8-2,59-61$ & 30 & $\mathrm{~T}$ & $T$ & 20 & 20 & is & 15 \\
\hline $8-3,23-25$ & 25 & - & $T$ & 25 & 20 & 15 & 15 \\
\hline $8-3,46-48$ & 20 & - & - & 30 & 25 & 5 & 20 \\
\hline $8-4,40-42$ & 25 & - & - & 30 & 20 & 10 & 15 \\
\hline $9-1,60-62$ & 30 & $\mathrm{~T}$ & - & 20 & 25 & 15 & 10 \\
\hline $9 \cdot 2,27-29$ & 25 & $\mathrm{~T}$ & ? & 25 & 25 & 10 & 15 \\
\hline $9-3,40-42$ & 15 & - & $\mathrm{T}$ & 30 & 30 & 5 & 20 \\
\hline $9-4,16-18$ & 10 & - & $\mathrm{T}$ & 15 & 60 & $\mathrm{~T}$ & 15 \\
\hline $10-1,137-139$ & 15 & - & $\mathrm{T}$ & 10 & 55 & $\mathrm{~T}$ & 20 \\
\hline $12-1,80-82$ & 15 & - & - & 70. & - & 10 & 5 \\
\hline $12-2,50-52$ & 15 & - & - & 75 & $T$ & 10 & $\mathrm{~T}$ \\
\hline $13-1,98-100$ & 15 & - & - & 50 & $\mathrm{~T}$ & 25 & 10 \\
\hline $13-2,60-62$ & 15 & - & - & 50 & ? & 25 & 10 \\
\hline $13-3,56-58$ & 15 & - & - & 50 & - & 25 & 10 \\
\hline $14-1,50-52$ & 15 & - & - & 50 & $\mathrm{~T}$ & 25 & 10 \\
\hline $14-2,50-52$ & 15 & - & - & 50 & $?$ & 25 & 10 \\
\hline $15-1,50-52$ & 15 & - & - & 50 & $\mathrm{~T}$ & 25 & 10 \\
\hline $15-2,50-52$ & 15 & - & - & 75 & - & 10 & $\mathrm{~T}$ \\
\hline
\end{tabular}

TABLE 14

Clay Mineralogy Data, Site $375^{\mathrm{a}}(<2 \mu \mathrm{m}$, carbonate free fraction)

\begin{tabular}{|c|c|c|c|c|c|c|c|}
\hline $\begin{array}{c}\text { Sample } \\
\text { (Interval in } \mathrm{cm} \text { ) }\end{array}$ & Illite & $\begin{array}{c}\text { Mixed } \\
\text { Layer } \\
10-14 \text { M }\end{array}$ & $\begin{array}{c}\text { Mixed } \\
\text { Layer } \\
14 \mathrm{C}-14 \mathrm{M}\end{array}$ & $\begin{array}{l}\text { Smec- } \\
\text { tite }\end{array}$ & $\begin{array}{l}\text { Atta- } \\
\text { pulgite }\end{array}$ & $\begin{array}{c}\text { Chlo- } \\
\text { rite }\end{array}$ & Kaolinite \\
\hline $2-2,37-40$ & 5 & - & - & 80 & 10 & 5 & $T$ \\
\hline $2-3,131-133$ & ? & - & - & 100 & $?$ & $\mathrm{~T}$ & $\mathrm{~T}$ \\
\hline $2-4,3-5$ & 15 & - & - & 40 & 20 & 25 & T \\
\hline $4-1,113-115$ & 10 & - & $\mathrm{T}$ & 50 & 25 & 15 & T \\
\hline $4-2,65-67$ & 20 & - & T & 40 & 20 & 20 & $\mathrm{~T}$ \\
\hline $4-3,65-68$ & 15 & - & $\mathrm{T}$ & 50 & 20 & 15 & $\mathrm{~T}$ \\
\hline $4-4,74-77$ & 15 & - & $\mathrm{T}$ & 50 & 15 & 20 & $\mathrm{~T}$ \\
\hline $4-5,76-78$ & 15 & - & $\mathrm{T}$ & 55 & 15 & 15 & $\mathrm{~T}$ \\
\hline $4, C C$ & 15 & - & 5 & so & 15 & 15 & $\mathrm{~T}$ \\
\hline $5-1,50-52$ & 10 & - & 5 & 60 & 10 & 15 & - \\
\hline $5-2,43-45$ & $\mathrm{~T}$ & - & - & 50 & 25 & 25 & $\mathrm{~T}$ \\
\hline $5-3,40-43$ & 5 & - & $\mathrm{T}$ & 80 & 5 & 10 & - \\
\hline $5-4,97-99$ & 10 & $?$ & $\mathrm{~T}$ & 50 & 20 & 20 & $\mathrm{~T}$ \\
\hline $5-5,31-32$ & 10 & ? & 5 & 50 & 15 & 15 & 5 \\
\hline $5-6,98-100$ & 15 & T & $\mathrm{T}$ & 55 & 10 & 20 & $\mathbf{T}$ \\
\hline $6-1,58-61$ & 10 & $\bar{T}$ & $\overline{5}$ & $\begin{array}{l}60 \\
60\end{array}$ & 15 & 15 & $\begin{array}{l}T \\
T\end{array}$ \\
\hline $6-3,102-104$ & 5 & $\underline{T}$ & & $\begin{array}{l}60 \\
65\end{array}$ & $\begin{array}{l}10 \\
10\end{array}$ & 20 & $\begin{array}{l}\mathrm{T} \\
\mathrm{T}\end{array}$ \\
\hline $\begin{array}{l}6-4,142-145 \\
6-5,52-54\end{array}$ & $\begin{array}{r}5 \\
10\end{array}$ & $\overline{-}$ & $\begin{array}{l}\mathrm{T} \\
\mathrm{T}\end{array}$ & $\begin{array}{l}65 \\
65\end{array}$ & $\begin{array}{l}10 \\
10\end{array}$ & $\begin{array}{l}20 \\
15\end{array}$ & $\begin{array}{l}\mathrm{T} \\
\mathrm{T}\end{array}$ \\
\hline $6-6,34-36$ & 10 & - & $\mathrm{T}$ & 65 & 10 & 15 & $\mathrm{~T}$ \\
\hline $7-1,133-135$ & 5 & - & 5 & 55 & 15 & 20 & $\mathrm{~T}$ \\
\hline $7-2,101-103$ & 5 & - & - & 60 & 5 & 30 & - \\
\hline $7-3,90-92$ & 5 & - & - & 65 & 10 & 20 & $T$ \\
\hline $7-4,62-64$ & 5 & - & $\mathrm{T}$ & 70 & 10 & 15 & $\mathrm{~T}$ \\
\hline $7-5,67-68$ & 5 & - & $\mathrm{T}$ & 70 & 10 & 15 & $\mathrm{~T}$ \\
\hline $7-6,82-84$ & 5 & - & - & 60 & 5 & 30 & $?$ \\
\hline $8-1,30-34$ & 5 & - & - & 60 & 10 & 25 & $\mathrm{~T}$ \\
\hline $8-2,49-51$ & 10 & - & - & 65 & 10 & 10 & 5 \\
\hline $8-3,49-52$ & 5 & - & - & 65 & 10 & 20 & $\mathrm{~T}$ \\
\hline $8-4,62-65$ & 10 & - & $\mathrm{T}$ & 65 & 10 & 5 & 10 \\
\hline $8-5,69-72$ & 10 & - & $\mathrm{T}$ & 75 & 5 & 10 & $\mathrm{~T}$ \\
\hline $8-6,36-38$ & 5 & - & - & 80 & 5 & 5 & 5 \\
\hline $9-1,45-47$ & 10 & - & - & 70 & 10 & 5 & 5 \\
\hline $9-2,89-91$ & 5 & ? & - & 70 & 10 & 15 & $\mathrm{~T}$ \\
\hline $9-3,94-96$ & 5 & - & - & 55 & 15 & 20 & 5 \\
\hline $9-4,123-126$ & 5 & - & - & 60 & 10 & 25 & $T$ \\
\hline $9-5,22-24$ & 5 & - & - & 70 & 10 & 10 & 5 \\
\hline $9-6,80-82$ & 10 & - & - & 70 & 5 & 15 & $\mathrm{~T}$ \\
\hline $10-1,124-126$ & 10 & $?$ & - & 50 & 20 & 10 & 10 \\
\hline $10-2,39-42$ & 10 & $\mathrm{~T}$ & $\mathrm{~T}$ & 55 & 5 & 5 & 15 \\
\hline $10-2,80-82$ & 5 & - & - & 45 & 25 & 10 & 15 \\
\hline $10-3,40-42$ & 5 & T & $T$ & 80 & $\mathrm{~T}$ & $\mathrm{~T}$ & 15 \\
\hline $10-3,80-82$ & & - & - & 80 & $?$ & - & 20 \\
\hline $11-1,95-97$ & $\mathrm{~T}$ & - & - & 60 & 20 & 15 & 5 \\
\hline $11-1,104-106$ & 10 & - & - & 40 & 20 & 25 & 5 \\
\hline $13-1,120-121$ & 5 & T & $\mathrm{T}$ & 70 & $?$ & $\mathrm{~T}$ & 25 \\
\hline
\end{tabular}

aH. Chamley Lab, Marseille, France. 
TABLE 15

Clay Mineralogy Data, Site $376^{\mathrm{a}}(<2 \mu \mathrm{m} \xi$ carbonate free fraction)

\begin{tabular}{|c|c|c|c|c|c|c|c|}
\hline $\begin{array}{c}\text { Sample } \\
\text { (Interval in } \mathrm{cm} \text { ) }\end{array}$ & Illite & $\begin{array}{c}\text { Mixed } \\
\text { Layer } \\
10-14 \mathrm{M}\end{array}$ & $\begin{array}{c}\text { Mixed } \\
\text { Layer } \\
14 \mathrm{C}-14 \mathrm{M}\end{array}$ & $\begin{array}{c}\text { Smec- } \\
\text { tite }\end{array}$ & $\begin{array}{c}\text { Atta- } \\
\text { pulgite }\end{array}$ & $\begin{array}{l}\text { Chlo- } \\
\text { rite }\end{array}$ & Kaolinite \\
\hline $1-1,74-76$ & 10 & 5 & 5 & 70 & - & 5 & 5 \\
\hline $1-1,100-102$ & 5 & T & T & 80 & 5 & $\mathrm{~T}$ & 10 \\
\hline $1-2,95-97$ & 5 & T & $=$ & 80 & 5 & 5 & 5 \\
\hline $1-3,50-52$ & 5 & $\mathrm{~T}$ & T & 80 & 5 & 5 & 5 \\
\hline $1-4,50-52$ & $\mathrm{~T}$ & - & $\bar{x}$ & 80 & 10 & $\mathrm{~T}$ & 10 \\
\hline $1-5,106-108$ & 5 & $\overline{-}$ & T & 80 & 5 & 5 & 5 \\
\hline $1-6,144-146$ & 5 & $\mathrm{~T}$ & $\mathrm{~T}$ & 85 & $\mathrm{~T}$ & 5 & 5 \\
\hline $2-1,88-90$ & 5 & $T$ & $\mathrm{~T}$ & 80 & 5 & 5 & \\
\hline $\begin{array}{l}2-2,71-74 \\
2-3,108-110\end{array}$ & $\begin{array}{l}5 \\
5\end{array}$ & $\begin{array}{l}\mathrm{T} \\
\mathrm{T}\end{array}$ & $\begin{array}{l}\mathrm{T} \\
\mathrm{T}\end{array}$ & $\begin{array}{l}85 \\
85\end{array}$ & $\begin{array}{c}5 \\
T\end{array}$ & ${ }_{5}^{T}$ & $\begin{array}{l}5 \\
5\end{array}$ \\
\hline $2-4,57-59$ & 5 & $\mathrm{~T}$ & $\mathrm{~T}$ & 80 & 5 & $\mathrm{~T}$ & 10 \\
\hline $3-1,146-148$ & 5 & $T$ & $\mathrm{~T}$ & 80 & 5 & 5 & 5 \\
\hline $3-2,120-122$ & 5 & $T$ & $\mathrm{~T}$ & 80 & 5 & 5 & 5 \\
\hline $3-3,70-72$ & 5 & $T$ & T & 80 & 5 & 5 & 5 \\
\hline $3-4,45-47$ & 5 & $T$ & $T$ & 80 & 5 & 5 & 5 \\
\hline $3-5,107-109$ & 5 & $\mathrm{~T}$ & $\mathrm{~T}$ & 85 & $\mathrm{~T}$ & $\mathrm{~T}$ & 10 \\
\hline $\begin{array}{l}4-1,88-90 \\
4-2,85-87\end{array}$ & $\begin{array}{l}\mathrm{T} \\
5\end{array}$ & $T$ & $T$ & 85 & 5 & $T$ & 10 \\
\hline & & $\begin{array}{l}\mathrm{T} \\
-\end{array}$ & $\begin{array}{l}T \\
T\end{array}$ & 80 & 5 & $\mathrm{~T}$ & \\
\hline $\begin{array}{l}5-1,100-102 \\
5-2,85-87\end{array}$ & $\begin{array}{r}5 \\
10\end{array}$ & ? & $\begin{array}{l}\mathrm{T} \\
\mathrm{T}\end{array}$ & $\begin{array}{l}80 \\
70\end{array}$ & $\begin{array}{l}5 \\
5\end{array}$ & $\begin{array}{l}\mathrm{T} \\
\mathrm{T}\end{array}$ & $\begin{array}{l}10 \\
15\end{array}$ \\
\hline $5-3,39-41$ & 5 & - & $\mathrm{T}$ & 70 & 10 & $\mathrm{~T}$ & 15 \\
\hline $5-3,125-127$ & 5 & - & $T$ & 70 & 10 & $\mathrm{~T}$ & 15 \\
\hline $5-4,80-82$ & 5 & - & T & 70 & 10 & $T$ & 15 \\
\hline $5-5,138-140$ & 5 & - & $\mathrm{T}$ & 70 & 10 & $\mathrm{~T}$ & 15 \\
\hline $\begin{array}{l}6-1,130-132 \\
6-2,80-82\end{array}$ & $\begin{array}{l}5 \\
5\end{array}$ & ${ }_{T}^{T}$ & $\begin{array}{l}\mathrm{T} \\
\mathrm{T}\end{array}$ & $\begin{array}{l}80 \\
80\end{array}$ & $\begin{array}{l}5 \\
5\end{array}$ & $\begin{array}{l}5 \\
5\end{array}$ & $\begin{array}{l}5 \\
5\end{array}$ \\
\hline $6-3,112-114$ & $\mathrm{~T}$ & - & - & 75 & 5 & $\vec{T}$ & 20 \\
\hline $6-4,44-46$ & 5 & - & - & 45 & 35 & 5 & 10 \\
\hline $7-1,100-102$ & $\mathrm{~T}$ & - & - & 50 & 30 & 15 & 5 \\
\hline $7-2,53-55$ & 5 & - & - & 50 & 30 & 15 & $\mathrm{~T}$ \\
\hline $7-2,85-87$ & 5 & - & - & 70 & 10 & 15 & $\mathrm{~T}$ \\
\hline $8-1,93-95$ & 5 & - & $\bar{z}$ & 55 & 25 & 15 & $\mathrm{~T}$ \\
\hline $\begin{array}{l}8-2,46-48 \\
8-3,125-127\end{array}$ & $\begin{array}{c}\mathrm{T} \\
10\end{array}$ & $\bar{z}$ & $\bar{z}$ & $\begin{array}{l}60 \\
70\end{array}$ & $\begin{array}{l}20 \\
10\end{array}$ & $\begin{array}{l}20 \\
10\end{array}$ & $\bar{T}$ \\
\hline $9-1,97-99$ & 5 & - & - & 60 & 20 & 15 & $?$ \\
\hline $9-2,60-62$ & T & - & - & 85 & 10 & 5 & $\dot{\mathrm{T}}$ \\
\hline $9-3,57-59$ & $\mathrm{~T}$ & - & - & 90 & 10 & $\mathrm{~T}$ & T \\
\hline $9-4,25-27$ & 5 & - & - & 55 & 25 & 15 & ? \\
\hline $10-1,83-85$ & $\mathrm{~T}$ & - & $\bar{x}$ & 60 & 25 & 15 & $T$ \\
\hline $10-2,65-67$ & $\mathrm{~T}$ & - & $\mathrm{T}$ & 60 & 25 & 15 & $\mathrm{~T}$ \\
\hline $10-3,108-110$ & 5 & - & - & 60 & 20 & 15 & $\mathrm{~T}$ \\
\hline $11-1,136-138$ & T & - & - & 60 & 25 & 15 & $\mathrm{~T}$ \\
\hline $\begin{array}{l}11-2,67-69 \\
11-3,44-46\end{array}$ & 5 & - & - & 60 & 20 & 15 & ? \\
\hline $11-3,44-46$ & 5 & - & - & 55 & 20 & 20 & $\mathrm{~T}$ \\
\hline $12-1,95-97$ & $T$ & - & - & 90 & 5 & 5 & $?$ \\
\hline $12-2,54-56$ & $\mathrm{~T}$ & - & - & 95 & 5 & $T$ & - \\
\hline & $\mathrm{T}$ & $\bar{z}$ & - & 95 & 5 & $\mathrm{~T}$ & - \\
\hline $12-5,67-69$ & $?$ & - & & 95 & 5 & T & - \\
\hline $13-1,135-137$ & - & - & - & 95 & 5 & $T$ & - \\
\hline $13-2,73-75$ & - & - & - & 100 & $T$ & T & - \\
\hline $13-3,106-108$ & - & - & - & 100 & $\mathrm{~T}$ & T & - \\
\hline $13-4,98-100$ & - & - & - & 100 & T & T & - \\
\hline $14, \mathrm{CC}$ & - & - & - & 100 & $\mathrm{~T}$ & $\mathrm{~T}$ & - \\
\hline $15-1,108-110$ & $\mathrm{~T}$ & - & - & 85 & 10 & 5 & $?$ \\
\hline $\begin{array}{l}15-2,78-80 \\
15-3,66-69\end{array}$ & $\begin{array}{l}\mathrm{T} \\
?\end{array}$ & $\overline{-}$ & $\bar{z}$ & $\begin{array}{l}65 \\
85\end{array}$ & $\begin{array}{l}25 \\
10\end{array}$ & $\begin{array}{r}10 \\
5\end{array}$ & $\begin{array}{l}\mathrm{T} \\
?\end{array}$ \\
\hline $16-1,68-70$ & $\mathrm{~T}$ & - & - & 85 & 10 & 5 & $\mathrm{~T}$ \\
\hline $17-1,116-118$ & $\mathrm{~T}$ & - & - & 75 & 15 & 10 & $\mathrm{~T}$ \\
\hline $18-1,106-108$ & ? & - & - & 75 & 15 & 10 & $\mathrm{~T}$ \\
\hline
\end{tabular}

aH. Chamley Lab, Marseille, France.
TABLE 16

Clay Mineralogy Data, Site $377^{\mathrm{a}}(<2 \mu \mathrm{m}$, carbonate free fraction)

\begin{tabular}{lccccccc}
\hline $\begin{array}{c}\text { Sample } \\
\text { (Interval in cm) }\end{array}$ & Illite & $\begin{array}{c}\text { Mixed } \\
\text { Layer } \\
10-14 \mathrm{M}\end{array}$ & $\begin{array}{c}\text { Mixed } \\
\text { Layer } \\
10-14 \mathrm{C}\end{array}$ & $\begin{array}{c}\text { Smec- } \\
\text { tite }\end{array}$ & $\begin{array}{c}\text { Atta- } \\
\text { pulgite }\end{array}$ & $\begin{array}{c}\text { Chlo- } \\
\text { rite }\end{array}$ & Kaolinite \\
\hline $1-1,89-91$ & 43 & - & - & 10 & - & 17 & 30 \\
$1-2,67-69$ & 45 & - & - & 14 & - & 18 & 23 \\
$2-1,102-105$ & 44 & - & - & 17 & - & 14 & 25 \\
$3-1,141-143$ & 26 & - & - & 28 & - & 16 & 30 \\
$4-1,84-86$ & 54 & - & - & 15 & - & 20 & 11 \\
$4-1,117-118$ & 25 & 13 & - & 19 & - & 11 & 28 \\
ap. Rouge CFP Lab, Bordeaux, France. & & & & & \\
\hline
\end{tabular}

TABLE 17

Clay Mineralogy Data, Site $378^{a}(<2 \mu \mathrm{m}$, carbonate free fraction)

\begin{tabular}{cccccccc}
\hline & & Mixed & Mixed & & & & \\
Sample & & Layer & Layer & Smec- & Atta- & Chlo- \\
(Interval in cm) & Illite & $10-14 \mathrm{M}$ & $10-14 \mathrm{C}$ & $\begin{array}{c}\text { tite } \\
\text { pulgite }\end{array}$ & $\begin{array}{c}\text { rite } \\
\text { Kaolinite }\end{array}$ \\
\hline
\end{tabular}

Hole 378

$\begin{array}{llllllll}1-1,110-112 & 52 & - & - & 16 & - & 17 & 15 \\ 1-2,40-42 & 41 & - & - & 18 & - & 18 & 23\end{array}$

$2-2,50-52$

$2-3,70-71$

$2-4,50-52$

$2-5,50-52$

3-1, 120-122

$3-2,70-72$

3-3, 50-52

$5-1,147-150$

$5-2,50-52$

$6-2,59-60$

6-3, 60-62

$7-1,50-52$

$7-2,50-52$

7-3, 89-91

$7-4,50-52$

$7-5,50-52$

8-1, 50-52

$8-2,80-82$

$11-3,60-62$

$11-4,53-55$

Hole 378 A

$1-1,120-122$

$1-2,70-72$

$1-3,70-72$

$1-4,49-51$

$1-5,70-72$

3-1, 56-58

3-2, 73-76

3-3, $71-74$

$3-4,58-61$

$3-5,41-43$

$3-6,71-73$

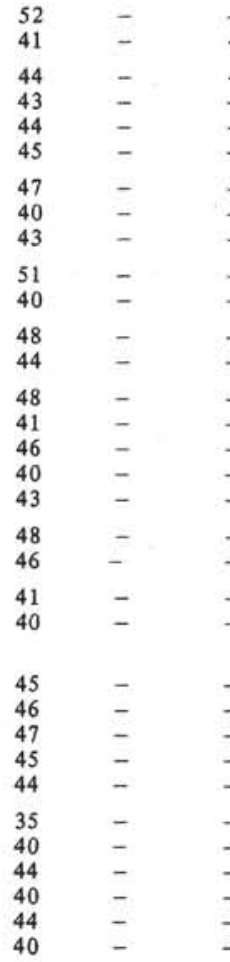

aP. Rouge CFP Lab, Bordeaux, France. 